\title{
ANÁLISIS ESTRUCTURAL DE UN BOSQUE MESÓFILO DE MONTAÑA EN EL EXTREMO ORIENTAL DE LA SIERRA MADRE DEL SUR (OAXACA), MÉXICO
}

\author{
Nancy R. Mejía-Domínguez', Jorge A. Meave'1, ${ }^{3}$ y Carlos A. Ruiz-Jiménez ${ }^{2}$ \\ ${ }^{1}$ Departamento de Ecología y Recursos Naturales, Facultad de Ciencias, Universidad Nacional \\ Autónoma de México, México 04510, D.F., México \\ ${ }^{2}$ Departamento de Biología Evolutiva, Facultad de Ciencias, Universidad Nacional Autónoma de \\ México, México 04510, D.F., México \\ ${ }^{3}$ Autor para la correspondencia. Correo-e: jamdc@hp.fciencias.unam.mx
}

\begin{abstract}
Resumen: Se analizó la estructura de la vegetación en una parcela de una hectárea de bosque mesófilo de montaña en Santo Tomás Teipan (Oaxaca, México). Considerando su ubicación marginal en el borde de una región de este tipo de vegetación en la Sierra Madre del Sur, se examinó la pregunta de si esta comunidad difería notablemente de otros bosques mesófilos propios de condiciones más adecuadas para esta formación vegetal. Cada individuo con diámetro a la altura del pecho (DAP) $\geq 2.5 \mathrm{~cm}$ fue ubicado en un sistema de coordenadas; además, se anotó su identidad taxonómica y se le midió la cobertura de la copa, el DAP, y las siguientes alturas: total, a la primera ramificación y a la base de la copa. Los valores obtenidos de área basal $\left(46.41 \mathrm{~m}^{2}\right.$ ha-1), cobertura $\left(44,247.33 \mathrm{~m}^{2} \mathrm{ha}^{-1}\right)$ y densidad $(1,035$ ind. ha-1) coinciden con los intervalos reportados para los bosques lluviosos montanos bajos ("lower montane rain forests") de América tropical. Se diferenciaron dos estratos por su estructura y composición, uno inferior (2 a $11 \mathrm{~m})$ y otro superior $(11$ a $30 \mathrm{~m})$. La dominancia general correspondió a Cornus disciflora, especie del dosel superior. En la parcela se distinguieron dos condiciones topográficas: ladera baja de cañada y cima, a las que parecen asociarse características edáficas contrastantes. A pesar de que esta heterogeneidad topográfica contrasta con la homogeneidad estructural del bosque, se detectaron patrones agregados para algunas especies distribuidas preferencialmente en alguna de dichas condiciones. En términos estructurales, el bosque de Teipan es comparable con otros bosques similares, pero su diversidad es relativamente baja. Esto puede deberse a la ausencia de taxa presentes en las comunidades secas tropicales adyacentes, a diferencia de otros bosques mesófilos cuya flora se enriquece con elementos de las comunidades tropicales húmedas con las que colindan.

Palabras clave: condiciones ecológicas marginales, Cornus disciflora, diversidad vegetal, estratificación del dosel, estructura de la vegetación.
\end{abstract}

\begin{abstract}
We analyzed vegetation structure in a 1-ha plot of cloud forest at Santo Tomás Teipan (Oaxaca, Mexico). Considering its marginal location near the edge of a region covered by this vegetation type on the Sierra Madre del Sur, we examined to what extent this community differed from other cloud forest communities thriving under better conditions for this plant formation. Each individual with diameter at breast height $(\mathrm{DBH}) \geq 2.5 \mathrm{~cm}$ was located in a system of coordinates; besides, its taxonomic identity, crown cover, DBH and total height as well as the height to the first branch and the crown base were recorded. Basal area $\left(46.41 \mathrm{~m}^{2} \mathrm{ha}^{-1}\right)$, cover $\left(44,247.33 \mathrm{~m}^{2} \mathrm{ha}^{-1}\right)$, and density $\left(1,035\right.$ ind. ha $\left.{ }^{-1}\right)$ figures fall within the known range for the lower montane rain forests of tropical America. According to structure and composition, two strata were differentiated: a lower one (2 to $11 \mathrm{~m})$, and an upper one (11 to $30 \mathrm{~m}$ ). Overall dominance corresponded to Cornus disciflora, an upper stratum species. In the plot two topographic conditions were distinguished, namely lower ravine slope and hilltop, which seem to be associated to contrasting soil characteristics. Although this topographic heterogeneity does not match the structural homogeneity of the forest, we detected some clumped patterns for individual species that were preferentially distributed in one of these conditions. The structure of the forest at Teipan is comparable to that of similar forests, but its diversity is relatively low. This may be explained by the absence of taxa occurring in the adjacent dry tropical communities, unlike other cloud forests whose flora appears to be enriched with some elements typical of tropical moist communities with which they abut.
\end{abstract}

Key words: canopy stratification, Cornus disciflora, marginal ecological conditions, plant diversity, vegetation structure.

$\mathbf{L}$ as comunidades vegetales que se desarrollan bajo la influencia de niebla frecuente en las montañas del trópico húmedo, agrupadas bajo el término general "bosques tropicales montanos de niebla" (Hamilton et al., 1995; Kappelle, 1996), constituyen una compleja transi- ción entre las comunidades tropicales de tierras bajas, más termófilas, y las comunidades propias de ambientes templados localizadas a mayor altitud (Beard, 1955; Whitmore, 1975; Richards, 1996). Esta transición depende de la interacción de los factores históricos, climáticos, edá- 
ficos y orográficos que operan en cada región, los que a su vez interactúan con factores locales como la orientación, la pendiente y la altitud. La variabilidad de estas condiciones entre sitios se refleja en la diversidad de bosques tropicales montanos de niebla alrededor del mundo (Hamilton et al., 1995).

En México estos bosques, agrupados bajo el término "bosque mesófilo de montaña" (Rzedowski, 1978), se distribuyen de forma discontinua en las principales formaciones montañosas del país. En la Sierra Madre del Sur, la superfice relativamente pequeña que cubren y el difícil acceso son quizá las causas de que los bosques mesófilos de esta región estén poco estudiados. Sólo se dispone de información estructural para bosques ubicados en las porciones noroccidental y central de esta sierra (Meave et al., 1992; Santiago y Jardel, 1993; Sánchez-Rodríguez et al., 2003), mientras que para la parte suroriental (estado de Oaxaca) los estudios se han enfocado a los aspectos florísticos (CamposVillanueva y Villaseñor, 1995; Acosta, 1997).

En las cercanías del Istmo de Tehuantepec, aproximadamente en el meridiano $96^{\circ} \mathrm{O}$, se encuentra el borde oriental de una de las áreas de bosque mesófilo de la Sierra Madre del Sur. En esta zona se localiza Santo Tomás Teipan, sitio de estudio de la presente investigación. Esta

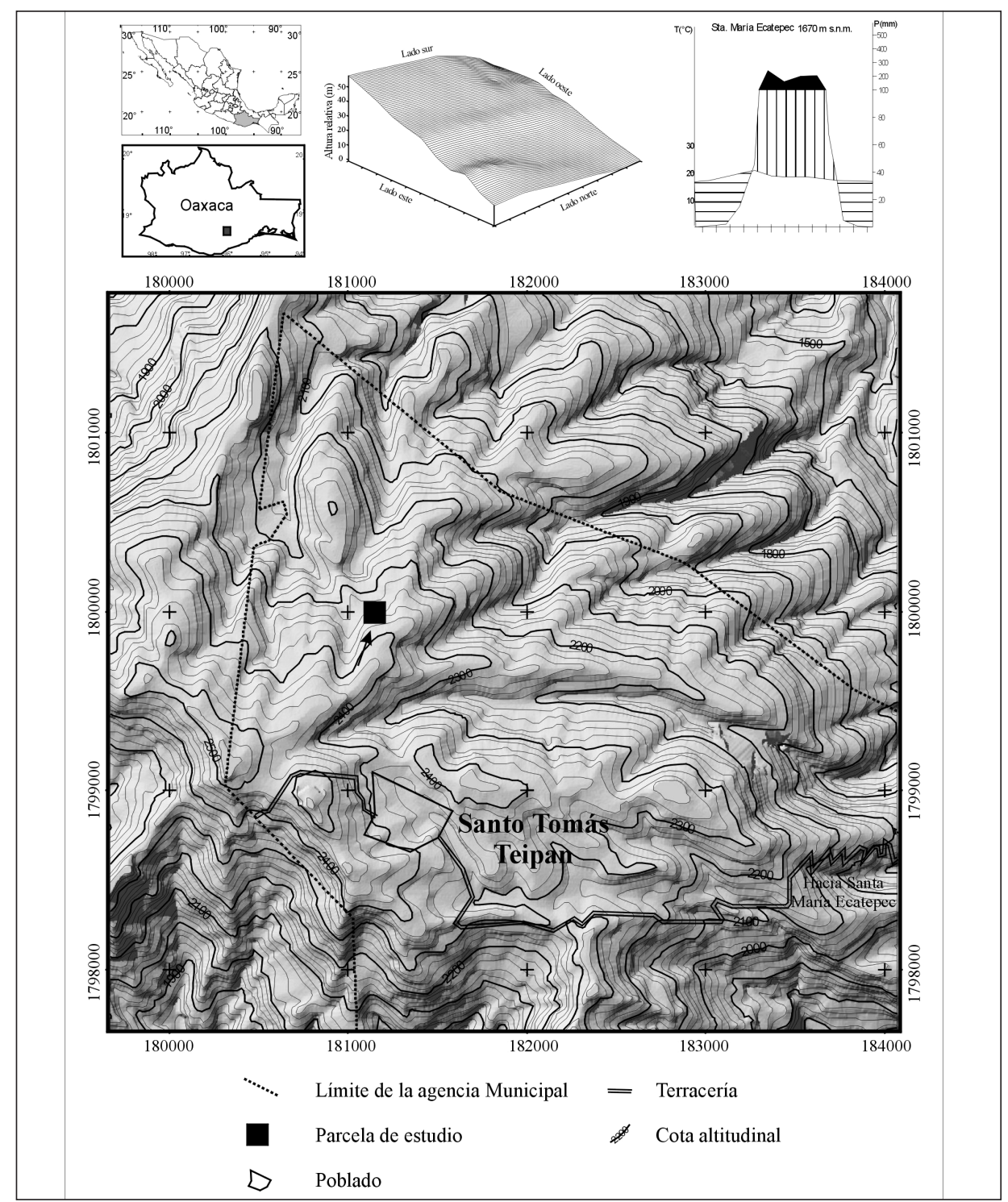

Figura 1. Localización de Santo Tomás Teipan y ubicación de la parcela de estudio. Las coordenadas UTM (zona 14) para la esquina noroeste son: 81,$185 ; 1,799,795$. 
ubicación marginal condujo a la pregunta de en qué medida este bosque difiere en su estructura y composición de otros localizados hacia el centro de las áreas de distribución de esta formación vegetal. Las peculiaridades de este bosque surgieron a partir de una comparación con otros bosques húmedos de montaña, con base en datos obtenidos en parcelas de una hectárea (Meave et al., 1992; Valencia y Jørgensen, 1992; Madsen y Øllgaard, 1993; Jørgensen et al., 1995; Valencia, 1995; Lieberman et al., 1996; Smith y Killeen, 1998; Arellanes-C., 2000).

\section{Área y parcela de estudio}

La investigación se realizó en los terrenos comunales de Santo Tomás Teipan (Mpio. de Santa María Ecatepec, Distrito de Yautepec), Oaxaca, en el sur de México (16 ${ }^{\circ} 14^{\prime}$ - $16^{\circ} 16^{\prime} \mathrm{N} ; 9^{\circ} 57^{\prime}-96^{\circ} 00^{\prime} \mathrm{O} ; 2,400$ m s.n.m.; figura 1 ). La geología regional se caracteriza por la presencia de rocas metamórficas precámbricas y paleozoicas, calizas cretácicas y rocas ígneas terciarias (INEGI, 1989; Ferrusquía-Villafranca, 1998). Los suelos más comunes son Acrisol húmico, Litosol y Regosol eútrico (Anónimo, 1981). La precipitación anual rebasa los 1,400 mm y la temperatura media anual, calculada a partir del gradiente térmico, es de $12.3^{\circ} \mathrm{C}$, cifra coincidente con la isoterma de $12^{\circ} \mathrm{C}$ indicada en las cartas climáticas (IG-UNAM, 1970; INEGI, 1984; García de Miranda, 1989). El tipo de clima para esta zona es $\mathrm{C}\left(\mathrm{w}_{2}\right)(\mathrm{w})$ big, es decir, templado subhúmedo, el más húmedo de los subhúmedos, con lluvias en verano e isotermal (IG-UNAM, 1970; figura 1). El bosque mesófilo de montaña cubre apenas 326.84 ha en la zona y se distribuye altitudinalmente entre 2,200 y $2,500 \mathrm{~m}$; a su alrededor se desarrollan encinares secos y, a elevaciones menores, una selva baja caducifolia (C.A. Ruiz-Jiménez, datos no publicados).

En este estudio se utilizó una parcela de una hectárea, ya que numerosas descripciones cuantitativas de bosques tropicales y de montaña se han basado en unidades de muestreo de este tamaño, lo cual facilita las comparaciones (Alder y Synnott, 1992). La parcela se ubica aprox. a $1 \mathrm{~km}$ en línea recta al norte de Santo Tomás Teipan, en la parte baja de la ladera noroeste del cerro Calabazo. El punto más bajo de la hectárea, la esquina NO, se ubica a 2,300 m de altitud, $60 \mathrm{~m}$ más abajo del punto más alto de la parcela (figura 1).

\section{Materiales y métodos}

En el muestreo de la vegetación se incluyeron todos los individuos enraizados dentro de la parcela con un diámetro a la altura del pecho (DAP) $\geq 2.5 \mathrm{~cm}$. Para ellos se registró su: identidad taxonómica, altura total, altura a la primera ramificación, altura a la base de la copa, diámetro a la altura del pecho (DAP), cobertura de la copa y localización en la parcela, usando un sistema de coordenadas. Las alturas se midieron con un medidor de distancias láser (Leica DISTO Basic). Con estos datos se calculó: el área basal (AB), la cobertura (C), la densidad (D), la frecuencia (F) y el valor de importancia relativa (VIR) (Goldsmith et al., 1976; Matteucci y Colma, 1982). Estos cálculos se hicieron para la parcela completa, para cuadros de $100 \mathrm{~m}^{2}$, para categorías diamétricas y de altura, y para las especies por separado.

Se determinó la estructura diamétrica de la comunidad y la poblacional de las especies representadas por más de 30 individuos. La estructura vertical se analizó mediante la distribución de frecuencias por clases de altura, en cuyas categorías se analizó la distribución del área basal y la cobertura (Popma et al., 1988; Bongers, 2001). Con base en la distribución de frecuencias se definieron tres categorías gruesas de altura $(0-11 \mathrm{~m}, 11.1-30 \mathrm{~m}, \mathrm{y}>30 \mathrm{~m})$, en las cuales se evaluó la distribución de la densidad, la cobertura, el área basal y la composición. Finalmente, se elaboraron diagramas de perfil a partir de dos transectos perpendiculares de $100 \times 10 \mathrm{~m}$, ubicados en la parte central de la parcela.

El análisis de la estructura horizontal incluyó tres aspectos. El primero fue la descripción de la distribución horizontal de las variables estructurales en la parcela en cuadros de $10 \times 10 \mathrm{~m}$. En segundo lugar, se analizó la distribución espacial de las especies más abundantes $(\mathrm{N}>35)$, usando dos métodos: el primero consistió en la evaluación de la bondad del ajuste de los datos de densidad por cuadro de la especie a la distribución de Poisson con una prueba de $\chi^{2}$, con el cálculo subsecuente del cociente varianza/ media para precisar el patrón de distribución (Zar, 1999); el segundo método consistió en el cálculo del cociente de distancias punto-planta (Cox, 1993). Finalmente, el grado de asociación entre pares de especies se evaluó mediante dos pruebas, una con área y otra basada en distancias, a partir de tablas de contingencia y un análisis de vecindario (Krebs, 1978).

Para evaluar la relación entre los atributos estructurales de la vegetación y la heterogeneidad topográfica de la parcela, los cuadros de $100 \mathrm{~m}^{2}$ fueron clasificados por medio del método de Ward (van Tongeren, 1995), utilizando el programa STATISTICA 6.0 (STATSOFT INC., 1998). Las clasificaciones se hicieron usando tres variables calculadas para los cuadros de $100 \mathrm{~m}^{2}$ : (1) datos de presencia-ausencia de las especies, (2) la densidad de especies y (3) el logaritmo natural (ln) del área basal por especie. La diversidad de especies en la comunidad se evaluó con los índices de Berger-Parker (d) y de Shannon-Wiener, la uniformidad (E) de este último (Magurran, 1988) y el índice $\alpha$ de Fisher (Hayek y Buzas, 1997). Se construyó una curva de acumulación de especies-área con el programa EstimateS (Versión 6.0b1; Colwell, 2001), y a partir del ajuste de la curva a los modelos Chao 1, Chao 2, Jackknife, Michaelis-Menten y Coleman se hicieron estimaciones del 
número esperado de especies en un muestreo suficientemente grande.

\section{Resultados}

Sintesis estructural. Se registraron 1,035 individuos con un DAP $\geq 2.5 \mathrm{~cm}$ pertenecientes a 39 especies, a las cuales corresponde un área basal de $46.41 \mathrm{~m}^{2}$ y una cobertura de $44,247.33 \mathrm{~m}^{2}(442.47 \%)$. Más de $80 \%$ del área basal total y más de 70\% de la cobertura correspondió a sólo 295 indi- viduos $(28.5 \%)$, todos ellos con DAP $\geq 20 \mathrm{~cm}$. En contraste, la densidad se concentró en los individuos de las categorías inferiores de DAP, ya que más de $50 \%$ correspondió a individuos con DAP entre 2.5 y $10 \mathrm{~cm}$; esto se ve reflejado en la forma de "J" invertida de la distribución de las clases diamétricas de los individuos (figura 2). Los valores promedio $( \pm 1 \mathrm{DE})$ por cuadro de $100 \mathrm{~m}^{2}$ fueron: riqueza, $6.1 \pm 1.9$ especies (intervalo: 2 a 13 especies); cobertura, $442.47 \pm 232.76 \mathrm{~m}^{2}$ (intervalo: 71.50 a $1,260.87 \mathrm{~m}^{2}$ ); densidad, $10.3 \pm 4.2$ individuos (intervalo:

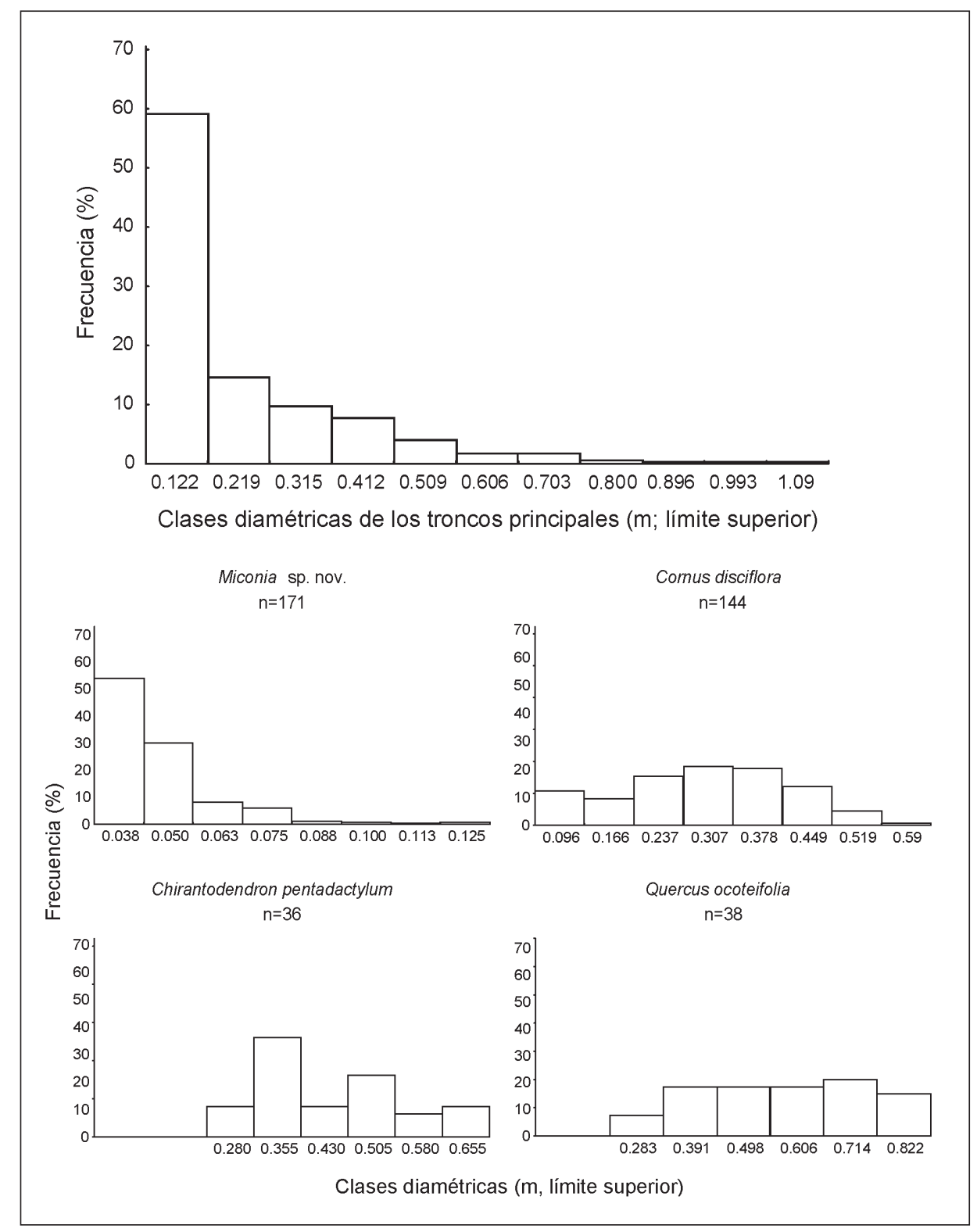

Figura 2. Distribución de frecuencias de las clases diamétricas de los troncos $(\mathrm{N}=1,035)$ y estructura poblacional de algunas especies. 
2 a 22 ind.); y área basal, $0.46 \pm 0.30 \mathrm{~m}^{2}$ (intervalo: 0.21 a $1.51 \mathrm{~m}^{2}$ ).

Valores de importancia relativa. De las 39 especies en la parcela, 11 tuvieron un VIR > 10, todas ellas con más de 30 individuos. El VIR más grande correspondió a Cornus disciflora (47.9), rebasando casi por 20 unidades al siguiente valor de importancia (Quercus ocoteifolia, 28.4). En contraste, $35 \%$ de las especies tuvieron un VIR $<1$. La figura 3a muestra algunas discontinuidades entre grupos de valor de importancia: (1) la especie dominante (Cornus disciflora), (2) un conjunto de 10 especies subordinadas pero estructuralmente importantes y (3) las 28 especies raras. En el caso de Cornus disciflora, el área basal hizo la mayor contribución porcentual al VIR, aunque los otros dos componentes también fueron importantes; por el contrario, para Miconia sp. nov. y Psychotria galeottiana, la contribución del área basal fue casi despreciable, por lo que sus respec-

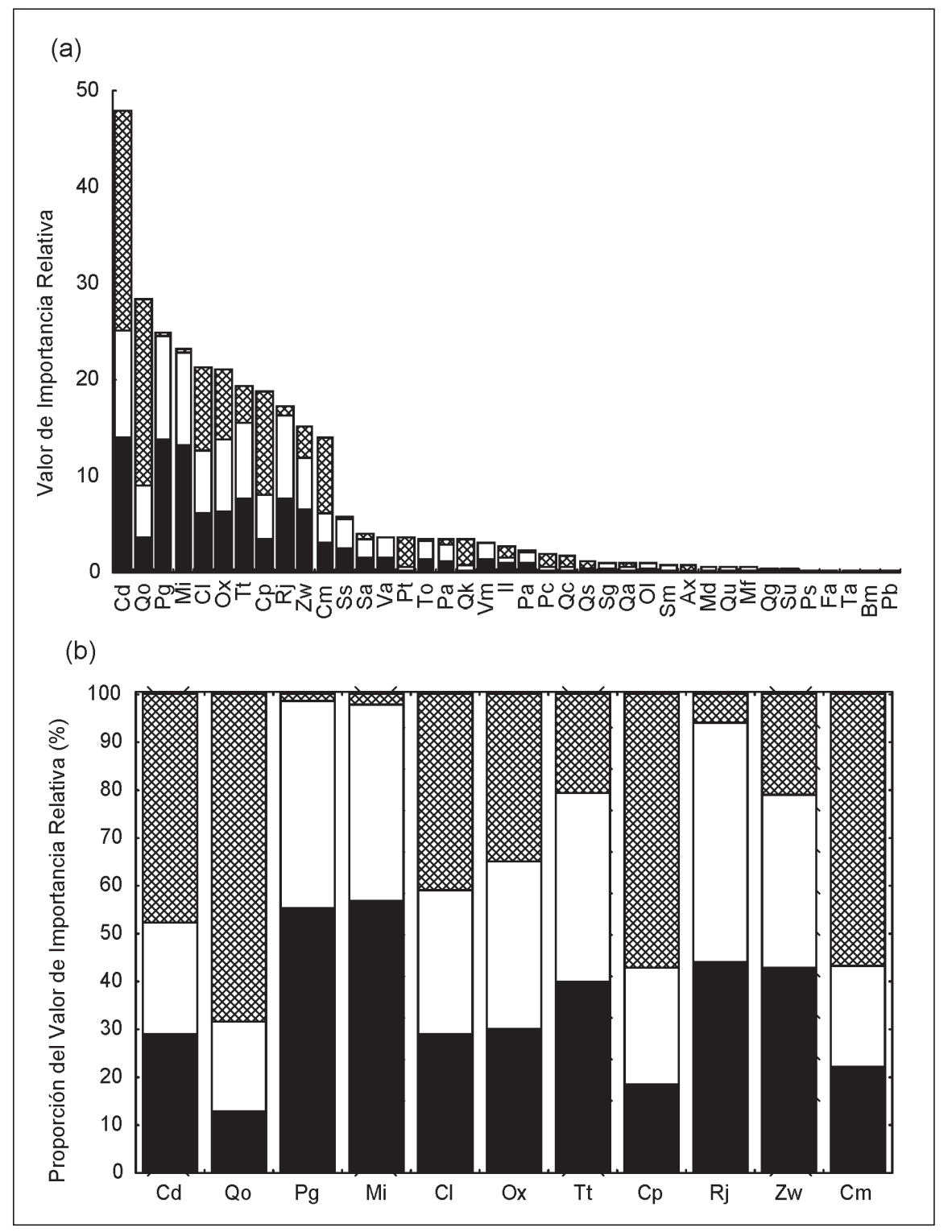

Figura 3. Gráficas de los valores de importancia relativa: (a) contribuciones absolutas de la densidad (barras negras), la frecuencia (barras blancas) y el área basal (barras tachadas) al VIR de cada especie; (b) contribuciones relativas de las mismas variables al VIR de la especie dominante y las diez especies subordinadas. Las abreviaturas de las especies se muestran en el cuadro 2. 
tivas posiciones (tercero y cuarto VIR) se debieron a sus densidades y frecuencias altas (figura 3b). En el caso de Quercus ocoteifolia, la contribución del área basal fue muy grande, lo que la hizo tener el segundo valor más alto de VIR, a pesar de su baja densidad.

Estructuras diamétricas. La distribución de las clases diamétricas de todos los individuos presentó forma de "J" invertida, es decir, la gran mayoría de ellos poseen diámetros pequeños (figura 2). El 12.9\% de estos individuos $(\mathrm{N}=133)$ son policaulescentes y en conjunto poseen $18 \%\left(8.37 \mathrm{~m}^{2}\right)$ del área basal total. Miconia sp. nov. fue la especie con la mayor proporción de individuos policaulescentes (18\% de su densidad). Las densidades de Miconia sp. nov. y Psychotria galeottiana se concentraron en las clases diamétricas pequeñas y su DAP máximo fue menor que $13 \mathrm{~cm}$. La distribución de frecuencias de clases diamétricas de Cornus disciflora fue unimodal, con la mayor frecuencia en una clase intermedia. Las poblaciones de Chiranthodendron pentadactylon y Quercus ocoteifolia

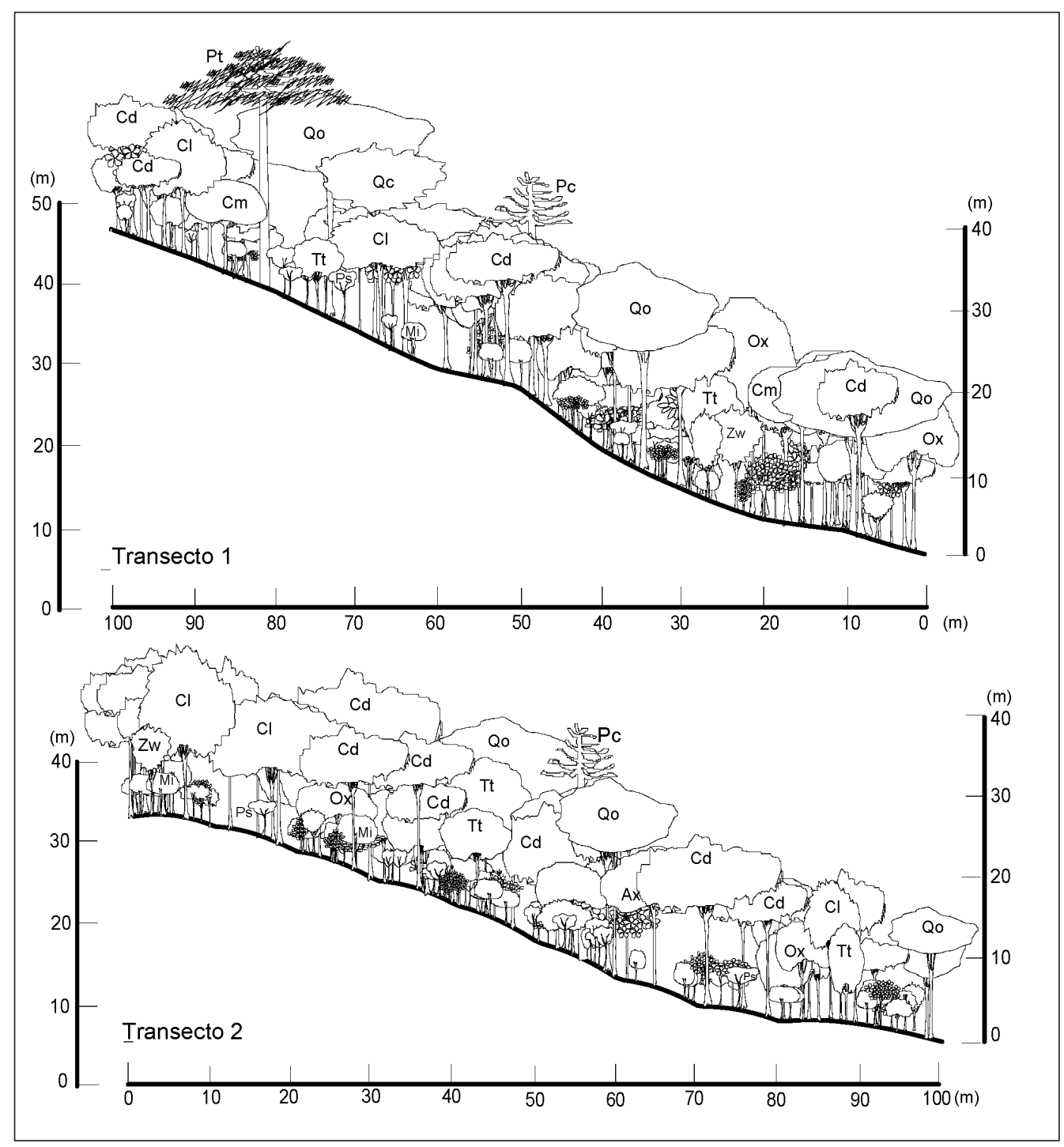

Figura 4. Diagramas de perfil de los transectos de $10 \times 100 \mathrm{~m}$ localizados en la parte central de la parcela. El transecto 1 está orientado de sur a norte y el transecto 2 de este a oeste. (Ax) Arbutus xalapensis, $(\mathrm{Cm})$ Clethra mexicana, $(\mathrm{Cl})$ Cleyera theaeoides, (Cd) Cornus disciflora, (Mi) Miconia sp. nov., (Ox) Oreopanax xalapensis, (Pc) Pinus chiapensis, (Pt) Pinus teocote, (Ps) Psychotria galeottiana, (Qo) Quercus ocoteifolia, (Tt) Ternstroemia tepezapote y (Zw) Zinowiewia sp. 
Bosque Mesófilo de MONTAÑa EN LA SierRa MAdRE DEL Sur

Cuadro 1. Distribución de las especies y de las variables estructurales en las tres categorías gruesas de altura. Se muestra el número total de especies por categoría, así como las especies exclusivas de cada una y las compartidas.

\begin{tabular}{|c|c|c|c|c|c|c|c|}
\hline \multirow{2}{*}{$\begin{array}{l}\text { Categoría } \\
\text { de altura }\end{array}$} & \multirow{2}{*}{$\begin{array}{l}\text { Altura } \\
(\mathrm{m})\end{array}$} & \multirow[t]{2}{*}{$\mathrm{N}$} & \multirow{2}{*}{$\begin{array}{c}\text { Cobertura } \\
\left(\mathrm{m}^{2}\right)\end{array}$} & \multirow{2}{*}{$\begin{array}{c}\text { Área } \\
\text { Basal }\left(\mathrm{m}^{2}\right)\end{array}$} & \multicolumn{3}{|c|}{ No. de especies } \\
\hline & & & & & total & compartidas & exclusivas \\
\hline I & $2-11$ & 601 & $5,770.82$ & 2.01 & 28 & 15 & 13 \\
\hline II & $11.1-30$ & 428 & $37,985.64$ & 43.54 & 25 & 17 & 8 \\
\hline III & $>30$ & 2 & 490.87 & 0.85 & 2 & 2 & 0 \\
\hline
\end{tabular}

Cuadro 2. Valores estructurales y valor de importancia relativa (VIR) para las 39 especies registradas en una parcela de una hectárea de bosque mesófilo en Teipan (Oaxaca), México. Se muestra el intervalo de alturas, i.e. altura máxima y mínima (IA), así como las categorías de altura (CA) en las que se encuentra cada especie. $\mathrm{D}=$ densidad; $\mathrm{F}=$ frecuencia; $\mathrm{AB}$ = área basal; $\mathrm{COB}=$ cobertura. En la parte superior de cada renglón se muestran los valores absolutos y en la inferior, entre paréntesis, los relativos; a partir de estos últimos se calculó el VIR.

\begin{tabular}{|c|c|c|c|c|c|c|c|c|}
\hline & Clave, especie y familia & $\begin{array}{l}\mathrm{IA} \\
(\mathrm{m})\end{array}$ & CA & $\mathrm{D}$ & $\mathrm{F}$ & $\begin{array}{l}\mathrm{AB} \\
\left(\mathrm{m}^{2}\right)\end{array}$ & $\begin{array}{l}\mathrm{COB} \\
\left(\mathrm{m}^{2}\right)\end{array}$ & VIR \\
\hline $\mathrm{Cd}$ & $\begin{array}{l}\text { Cornus disciflora DC. } \\
\text { [Cornaceae] }\end{array}$ & $2.5-26.0$ & I, II & $\begin{array}{c}144 \\
(13.9)\end{array}$ & $\begin{array}{c}68 \\
(11.1)\end{array}$ & $\begin{array}{l}10.63 \\
(22.9)\end{array}$ & $10,656.9$ & 47.9 \\
\hline Qo & $\begin{array}{l}\text { Quercus ocoteifolia Liebm. } \\
\text { [Fagaceae] }\end{array}$ & $15.0-32.0$ & II, III & $\begin{array}{c}38 \\
(3.7)\end{array}$ & $\begin{array}{c}32 \\
(5.2)\end{array}$ & $\begin{array}{c}9.04 \\
(19.4)\end{array}$ & $7,416.7$ & 28.4 \\
\hline Pg & $\begin{array}{l}\text { Psychotria galeottiana (M.Martens) } \\
\text { C.M. Taylor et Lorence [Rubiaceae] }\end{array}$ & $2.2-7.0$ & I & $\begin{array}{c}142 \\
(13.7)\end{array}$ & $\begin{array}{c}66 \\
(10.8)\end{array}$ & $\begin{array}{l}0.17 \\
(0.3)\end{array}$ & 509.4 & 24.9 \\
\hline Mi & $\begin{array}{l}\text { Miconia sp. nov. } \\
\text { [Melastomataceae] }\end{array}$ & $2.3-8.5$ & 1 & $\begin{array}{c}137 \\
(13.2)\end{array}$ & $\begin{array}{c}58 \\
(9.59)\end{array}$ & $\begin{array}{l}0.24 \\
(0.5)\end{array}$ & $1,125.6$ & 23.3 \\
\hline $\mathrm{Cl}$ & $\begin{array}{l}\text { Cleyera theaeoides (Sw.) Choisy } \\
\text { [Theaceae] }\end{array}$ & $3.0-23.5$ & I, II & $\begin{array}{c}64 \\
(6.2)\end{array}$ & $\begin{array}{c}39 \\
(6.4)\end{array}$ & $\begin{array}{l}4.03 \\
(8.7)\end{array}$ & $4,390.3$ & 21.2 \\
\hline Ox & $\begin{array}{l}\text { Oreopanax xalapensis (Kunth) Decne. } \\
\text { et Planch. [Araliaceae] }\end{array}$ & $4.4-27.0$ & I, II & $\begin{array}{l}66 \\
(6.4)\end{array}$ & $\begin{array}{c}45 \\
(7.4)\end{array}$ & $\begin{array}{l}3.45 \\
(7.4)\end{array}$ & $3,967.2$ & 21.2 \\
\hline $\mathrm{Tt}$ & $\begin{array}{l}\text { Ternstroemia tepezapote Schltdl. et } \\
\text { Cham. [Theaceae] }\end{array}$ & $2.5-22.0$ & I, II & $\begin{array}{c}80 \\
(7.7)\end{array}$ & $\begin{array}{c}47 \\
(7.7)\end{array}$ & $\begin{array}{l}1.84 \\
(4.0)\end{array}$ & $3,156.6$ & 19.4 \\
\hline Cp & $\begin{array}{l}\text { Chiranthodendron pentadactylon } \\
\text { Larreat. [Sterculiaceae] }\end{array}$ & $10.0-26.0$ & I, II & $\begin{array}{c}36 \\
(3.5)\end{array}$ & $\begin{array}{c}28 \\
(4.6)\end{array}$ & $\begin{array}{c}4.98 \\
(10.7)\end{array}$ & $2,960.0$ & 18.8 \\
\hline Rj & $\begin{array}{l}\text { Rapanea juergensenii Mez } \\
\text { [Myrsinaceae] }\end{array}$ & $2.4-17.0$ & I, II & $\begin{array}{l}79 \\
(7.6)\end{array}$ & $\begin{array}{c}53 \\
(8.7)\end{array}$ & $\begin{array}{l}0.46 \\
(1.0)\end{array}$ & $1,504.5$ & 17.3 \\
\hline $\mathrm{Zw}$ & $\begin{array}{l}\text { Zinowiewia sp. } \\
\text { [Celastraceae] }\end{array}$ & $3.7-28.0$ & I, II & $\begin{array}{c}67 \\
(6.5)\end{array}$ & $\begin{array}{c}33 \\
(5.4)\end{array}$ & $\begin{array}{l}1.48 \\
(3.2)\end{array}$ & $1,430.0$ & 15.1 \\
\hline $\mathrm{Cm}$ & $\begin{array}{l}\text { Clethra mexicana DC. } \\
\text { [Clethraceae] }\end{array}$ & $4.0-27.0$ & I, II & $\begin{array}{c}32 \\
(3.1)\end{array}$ & $\begin{array}{c}18 \\
(3.0)\end{array}$ & $\begin{array}{l}3.66 \\
(7.9)\end{array}$ & $1,587.5$ & 13.9 \\
\hline Sa & $\begin{array}{l}\text { Styrax argenteus C.Presl. } \\
\text { [Styracaceae] }\end{array}$ & $2.0-20.0$ & I, II & $\begin{array}{c}15 \\
(1.4)\end{array}$ & $\begin{array}{c}12 \\
(2.0)\end{array}$ & $\begin{array}{l}0.26 \\
(0.6)\end{array}$ & 603.1 & 4.0 \\
\hline Va & $\begin{array}{l}\text { Vallesia aurantiaca (M.Martens et } \\
\text { Galeotti) J.F.Morales [Apocynaceae] }\end{array}$ & $3.8-6.0$ & 1 & $\begin{array}{l}16 \\
(1.5)\end{array}$ & $\begin{array}{c}13 \\
(2.1)\end{array}$ & $\begin{array}{l}0.01 \\
(0.0)\end{array}$ & 68.9 & 3.7 \\
\hline Pt & $\begin{array}{l}\text { Pinus teocote Schltdl. et Cham. } \\
\text { [Pinaceae] }\end{array}$ & $30.0-35.0$ & II, III & $\begin{array}{c}2 \\
(0.2)\end{array}$ & $\begin{array}{c}2 \\
(0.3)\end{array}$ & $\begin{array}{l}1.44 \\
(3.1)\end{array}$ & 556.8 & 3.6 \\
\hline To & $\begin{array}{l}\text { Turpinia occidentalis (Sw.) G.Don } \\
\text { [Staphyleaceae] }\end{array}$ & $3.2-18.0$ & I, II & $\begin{array}{c}13 \\
(1.3)\end{array}$ & $\begin{array}{c}12 \\
(2.0)\end{array}$ & $\begin{array}{l}0.13 \\
(0.3)\end{array}$ & 311.1 & 3.5 \\
\hline $\mathrm{Pa}$ & $\begin{array}{l}\text { Persea americana Mill. } \\
\text { [Lauraceae] }\end{array}$ & $3.6-24.0$ & I, II & $\begin{array}{c}11 \\
(1.1)\end{array}$ & $\begin{array}{c}11 \\
(1.8)\end{array}$ & $\begin{array}{l}0.28 \\
(0.6)\end{array}$ & 352.4 & 3.5 \\
\hline Qk & $\begin{array}{l}\text { Quercus skinneri Benth. } \\
\text { [Fagaceae] }\end{array}$ & $20.0-28.0$ & II & $\begin{array}{c}3 \\
(0.3)\end{array}$ & $\begin{array}{c}3 \\
(0.5)\end{array}$ & $\begin{array}{l}1.24 \\
(2.7)\end{array}$ & 707.8 & 3.5 \\
\hline $\mathrm{Vm}$ & $\begin{array}{l}\text { Viburnum membranaceum (Oerst.) } \\
\text { Hemsl. [Caprifoliaceae] }\end{array}$ & $3.5-10.4$ & I & $\begin{array}{c}13 \\
(1.3)\end{array}$ & $\begin{array}{c}11 \\
(1.8)\end{array}$ & $\begin{array}{l}0.05 \\
(0.1)\end{array}$ & 133.5 & 3.2 \\
\hline II & $\begin{array}{l}\text { Ilex sp. } \\
\text { [Aquifoliaceae] }\end{array}$ & $2.8-25.0$ & I, II & $\begin{array}{c}9 \\
(0.9)\end{array}$ & $\begin{array}{c}4 \\
(0.7)\end{array}$ & $\begin{array}{l}0.56 \\
(1.2)\end{array}$ & 309.9 & 2.7 \\
\hline $\mathrm{Pa}$ & $\begin{array}{l}\text { Parathesis sp. } \\
\text { [Myrsinaceae] }\end{array}$ & $5.5-11.0$ & I & $\begin{array}{c}9 \\
(0.9)\end{array}$ & $\begin{array}{c}8 \\
(1.3)\end{array}$ & $\begin{array}{l}0.06 \\
(0.1)\end{array}$ & 182.2 & 2.3 \\
\hline
\end{tabular}


Nancy R. Mejía-Domínguez, Jorge A. Meave y Carlos A. Ruiz-Jiménez

Cuadro 2. Continuación

\begin{tabular}{|c|c|c|c|c|c|c|c|c|}
\hline & Clave, especie y familia & $\begin{array}{l}\mathrm{IA} \\
(\mathrm{m})\end{array}$ & CA & $\mathrm{D}$ & $\mathrm{F}$ & $\begin{array}{l}\mathrm{AB} \\
\left(\mathrm{m}^{2}\right)\end{array}$ & $\begin{array}{l}\mathrm{COB} \\
\left(\mathrm{m}^{2}\right)\end{array}$ & VIR \\
\hline Pc & $\begin{array}{l}\text { Pinus chiapensis (Martínez) Andresen } \\
\text { [Pinaceae] }\end{array}$ & $26.0-28.0$ & II & $\begin{array}{c}2 \\
(0.2)\end{array}$ & $\begin{array}{c}2 \\
(0.3)\end{array}$ & $\begin{array}{l}0.67 \\
(1.4)\end{array}$ & 145.3 & 2.0 \\
\hline Qc & $\begin{array}{l}\text { Quercus candicans Née } \\
\text { [Fagaceae] }\end{array}$ & $24.0-25.0$ & II & $\begin{array}{c}2 \\
(0.2)\end{array}$ & $\begin{array}{c}2 \\
(0.3)\end{array}$ & $\begin{array}{l}0.57 \\
(1.2)\end{array}$ & 605.7 & 1.7 \\
\hline Qs & $\begin{array}{l}\text { Quercus sapotiifolia Liebm. } \\
\text { [Fagaceae] }\end{array}$ & $18.0-24.0$ & II & $\begin{array}{c}2 \\
(0.2)\end{array}$ & $\begin{array}{c}1 \\
(0.2)\end{array}$ & $\begin{array}{l}0.37 \\
(0.8)\end{array}$ & 569.4 & 1.1 \\
\hline Sg & $\begin{array}{l}\text { Solandra guttata D. Don } \\
\text { [Solanaceae] }\end{array}$ & - & - & $\begin{array}{c}4 \\
(0.4)\end{array}$ & $\begin{array}{c}4 \\
(0.7)\end{array}$ & $\begin{array}{c}0.005 \\
(0.0)\end{array}$ & 0.0 & 1.1 \\
\hline Qa & $\begin{array}{l}\text { Quercus affinis Scheidw. } \\
\text { [Fagaceae] }\end{array}$ & $16.0-26.0$ & II & $\begin{array}{c}2 \\
(0.2)\end{array}$ & $\begin{array}{c}2 \\
(0.3)\end{array}$ & $\begin{array}{l}0.20 \\
(0.4)\end{array}$ & 123.7 & 1.0 \\
\hline Ol & $\begin{array}{l}\text { Oreopanax langlassei Standl. } \\
\text { [Araliaceae] }\end{array}$ & $3.5-5.8$ & I & $\begin{array}{c}4 \\
(0.4)\end{array}$ & $\begin{array}{c}3 \\
(0.5)\end{array}$ & $\begin{array}{l}0.01 \\
(0.0)\end{array}$ & 30.8 & 0.9 \\
\hline Sm & $\begin{array}{l}\text { Sapium sp. } \\
\text { [Euphorbiaceae] }\end{array}$ & $4.0-7.0$ & I & $\begin{array}{c}3 \\
(0.3)\end{array}$ & $\begin{array}{c}3 \\
(0.5)\end{array}$ & $\begin{array}{l}0.00 \\
(0.0)\end{array}$ & 16.3 & 0.8 \\
\hline Ax & $\begin{array}{l}\text { Arbutus xalapensis Kunth } \\
\text { [Ericaceae] }\end{array}$ & $18.0-18.8$ & II & $\begin{array}{c}1 \\
(0.1)\end{array}$ & $\begin{array}{c}1 \\
(0.2)\end{array}$ & $\begin{array}{l}0.24 \\
(0.5)\end{array}$ & 78.5 & 0.8 \\
\hline Md & $\begin{array}{l}\text { Meliosma dentata (Liebm.) Urb. } \\
\text { [Sabiaceae] }\end{array}$ & $4.0-12.0$ & I, II & $\begin{array}{c}2 \\
(0.2)\end{array}$ & $\begin{array}{c}2 \\
(0.3)\end{array}$ & $\begin{array}{l}0.02 \\
(0.1)\end{array}$ & 39.8 & 0.6 \\
\hline Qu & $\begin{array}{l}\text { Quercus uxoris McVaugh } \\
\text { [Fagaceae] }\end{array}$ & $5.3-16.0$ & I, II & $\begin{array}{c}2 \\
(0.2)\end{array}$ & $\begin{array}{c}2 \\
(0.3)\end{array}$ & $\begin{array}{l}0.02 \\
(0.0)\end{array}$ & 45.3 & 0.6 \\
\hline Mf & $\begin{array}{l}\text { Myrcianthes fragrans (Sw.) McVaugh } \\
\text { [Myrtaceae] }\end{array}$ & $4.1-5.5$ & 1 & $\begin{array}{c}2 \\
(0.2)\end{array}$ & $\begin{array}{c}2 \\
(0.3)\end{array}$ & $\begin{array}{l}0.00 \\
(0.0)\end{array}$ & 6.7 & 0.5 \\
\hline Qg & $\begin{array}{l}\text { Quercus glabrescens Benth. } \\
\text { [Fagaceae] }\end{array}$ & $14.0-14.0$ & II & $\begin{array}{c}1 \\
(0.1)\end{array}$ & $\begin{array}{c}1 \\
(0.2)\end{array}$ & $\begin{array}{l}0.09 \\
(0.2)\end{array}$ & 53.5 & 0.5 \\
\hline Su & $\begin{array}{l}\text { Saurauia sp. } \\
\text { [Actinidaceae] }\end{array}$ & $18.0-18.0$ & II & $\begin{array}{c}1 \\
(0.1)\end{array}$ & $\begin{array}{c}1 \\
(0.2)\end{array}$ & $\begin{array}{l}0.05 \\
(0.1)\end{array}$ & 44.2 & 0.4 \\
\hline Ps & $\begin{array}{l}\text { Prunus serotina var. capuli (Cav.) } \\
\text { McVaugh [Rosaceae] }\end{array}$ & $7.5-7.5$ & 1 & $\begin{array}{c}1 \\
(0.1)\end{array}$ & $\begin{array}{c}1 \\
(0.2)\end{array}$ & $\begin{array}{l}0.00 \\
(0.0)\end{array}$ & 22.5 & 0.3 \\
\hline $\mathrm{Fa}$ & $\begin{array}{l}\text { Fuchsia arborescens Sims } \\
\text { [Onagraceae] }\end{array}$ & $5.8-5.8$ & 1 & $\begin{array}{c}1 \\
(0.1)\end{array}$ & $\begin{array}{c}1 \\
(0.2)\end{array}$ & $\begin{array}{l}0.00 \\
(0.0)\end{array}$ & 38.5 & 0.3 \\
\hline Та & $\begin{array}{l}\text { Trichilia havanensis Jacq. } \\
\text { [Meliaceae] }\end{array}$ & $6.0-6.0$ & 1 & $\begin{array}{c}1 \\
(0.1)\end{array}$ & $\begin{array}{c}1 \\
(0.2)\end{array}$ & $\begin{array}{l}0.00 \\
(0.0)\end{array}$ & 2.7 & 0.3 \\
\hline $\mathrm{Bm}$ & $\begin{array}{l}\text { Berberis moranensis Schult. et Schult.f. } \\
\text { [Berberidaceae] }\end{array}$ & $2.5-2.5$ & 1 & $\begin{array}{c}1 \\
(0.1)\end{array}$ & $\begin{array}{c}1 \\
(0.2)\end{array}$ & $\begin{array}{l}0.00 \\
(0.0)\end{array}$ & 2.4 & 0.3 \\
\hline $\mathrm{Pb}$ & $\begin{array}{l}\text { Prunus brachybotrya Zucc. } \\
\text { [Rosaceae] }\end{array}$ & $3.0-3.0$ & 1 & $\begin{array}{c}1 \\
(0.1)\end{array}$ & $\begin{array}{c}1 \\
(0.2)\end{array}$ & $\begin{array}{l}0.00 \\
(0.0)\end{array}$ & 5.3 & 0.3 \\
\hline
\end{tabular}

estuvieron caracterizadas por la ausencia de individuos de tallas pequeñas (DAP $\leq 28 \mathrm{~cm}$ ), lo que sugiere una ausencia de incorporación reciente de individuos jóvenes (figura 2).

Estructura vertical. Los dos estratos definidos inicialmente están claramente diferenciados por su estructura y composición (figuras 4 y 5). El estrato inferior del dosel (2 a 11 m) está dominado por Psychotria galeottiana y Miconia sp. nov., y además su composición florística incluye principalmente a Berberis moranensis, Fuchsia arborescens, Myrcianthes fragans, Parathesis sp., Prunus brachybotria, Sapium sp., Trichilia havanensis, Vallesia aurantiaca y Viburnum membranaceum. El estrato superior (11 a 30 m) está constituido por Cornus disciflora, Quercus ocoteifolia, Ternstroemia tepezapote, Cleyera theaeoides y Clethra mexicana, y también presenta otras especies exclusivas como Arbutus xalapensis, Quercus affinis, Q. candicans,
Q. glabrescens, $Q$. sapotiifolia y $Q$. skinneri. En la parcela se registraron dos individuos emergentes, uno de Pinus chiapensis y el otro de Quercus ocoteifolia (cuadro 1 y 2).

Estructura horizontal. Los dos métodos utilizados para analizar la distribución espacial de las especies mostraron que pocas de ellas tienen distribuciones aleatorias. De acuerdo con el método con área, cinco especies presentaron un patrón agregado, mientras que el método sin área arrojó este resultado para ocho especies.

La asociación de Cleyera theaeoides con Zinowiewia sp. fue significativa de acuerdo a los dos métodos utilizados $\left(\chi^{2}=4.5\right.$ y $\chi^{2}=7.2$, respectivamente $)$; el método con área indicó una asociación negativa $(\mathrm{V}=-0.21)$. En las zonas altas de la parcela, donde se distribuye preferentemente Cleyera theaeoides, prácticamente no hay individuos de Zinowiewia sp. (figura 6a). De acuerdo con el método con 


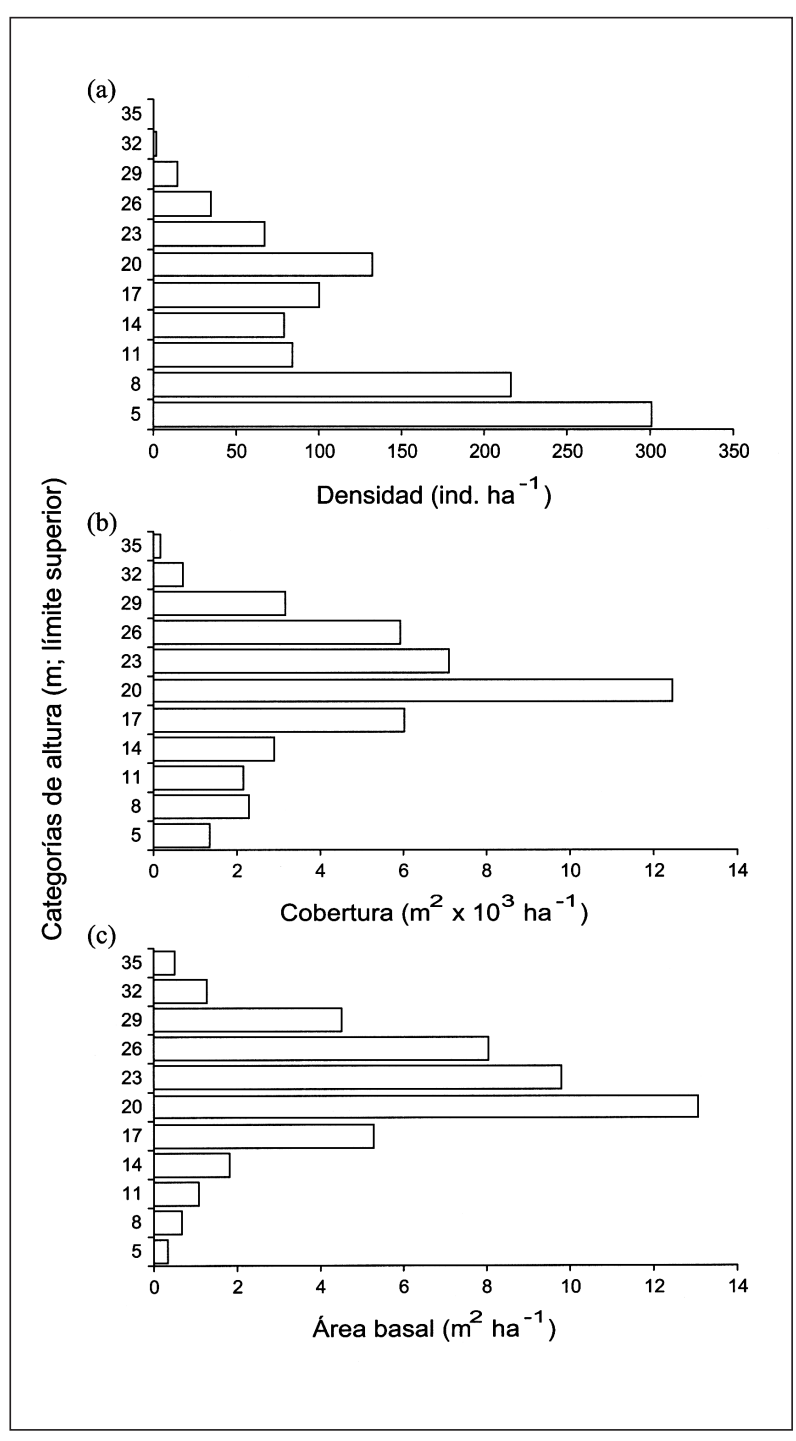

Figura 5. Distribución vertical de (a) la densidad, (b) la cobertura y (c) el área basal.

área, Psychotria galeottiana y Miconia sp. nov. (figura 6b) están asociadas positivamente $\left(\chi^{2}=8.2, \mathrm{~V}=0.29\right)$. La distribución espacial en la parcela de los grupos formados en los tres dendrogramas obtenidos con las clasificaciones de los cuadros no parece mostrar la existencia de una relación clara entre éstos y la topografía del terreno (figura 7).

Diversidad. Se registraron en total 39 especies pertenecientes a 29 géneros y a 26 familias (cuadro 2). El cuadro 3 muestra los índices calculados para los grupos formados al usar diferentes valores mínimos de DAP. El índice de Berger-Parker, que es un índice de dominancia, creció conforme aumentó el DAP mínimo, ya que la densi- (a) Cleyera theaeoides - Zinowieia $\mathrm{sp}$

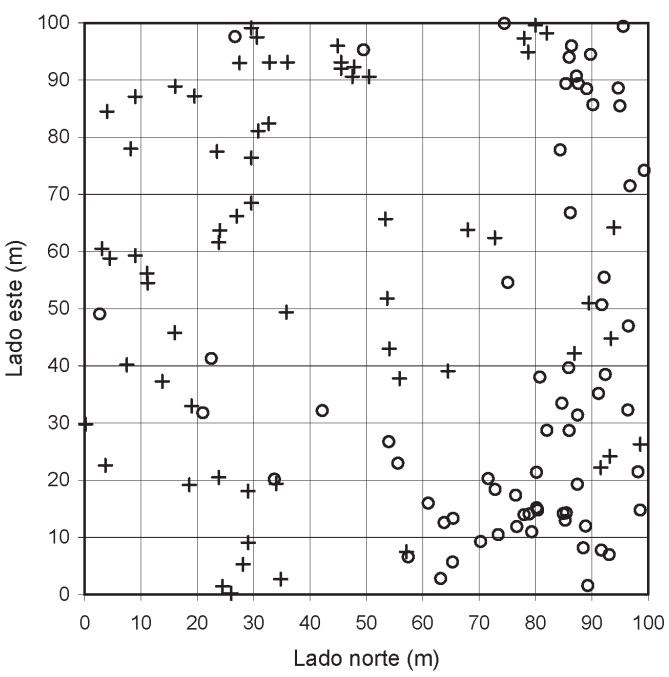

(b) Psychotria galeottiana - Miconia sp.nov.

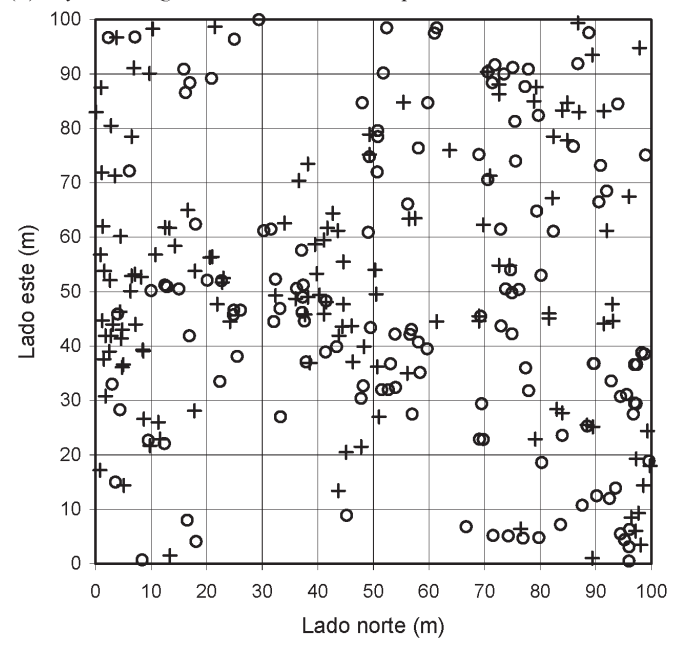

Figura 6. Distribución de pares de especies. (a) Cleyera theaeoides (+) y Zinowiewia sp. (o), (b) Psychotria galeottiana (o) y Miconia sp. nov. (+) que presentaron asociación positiva cuando se utilizó el método con área.

dad total disminuye notablemente, a diferencia de lo que sucede con la densidad de Cornus disciflora, la especie más abundante en todos esos grupos. En general, los valores de $\alpha$ de Fisher, H', H' máx y E disminuyeron conforme aumentó el DAP mínimo, pero el valor más alto se obtuvo usando un DAP $\geq 3.3 \mathrm{~cm}$. La curva de acumulación de especiesárea no alcanzó una asíntota, aunque la pendiente disminuyó claramente hacia el final de la curva (figura 8). El número de especies esperado según los estimadores de riqueza Michaelis-Menten, Jackknife y Coleman fluctuó entre 37.6, 47.9 y 38.9, respectivamente; de éstos, el estimador de Coleman resultó prácticamente idéntico al número de especies registradas en la parcela. Sin embargo, 
a)

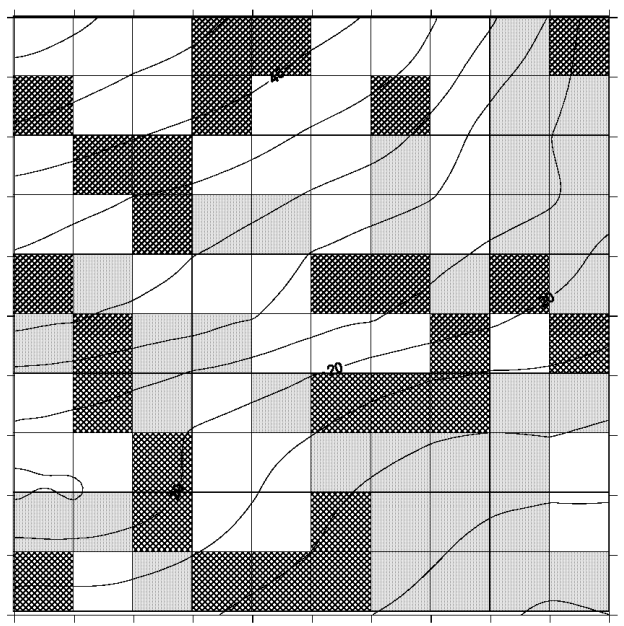

b)

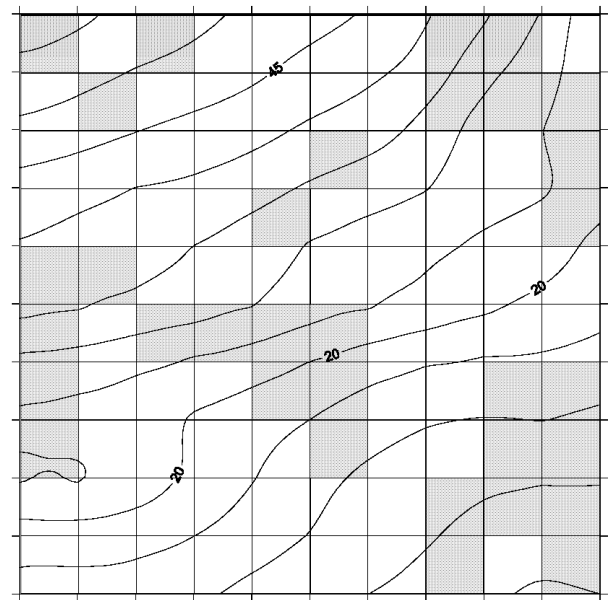

c)

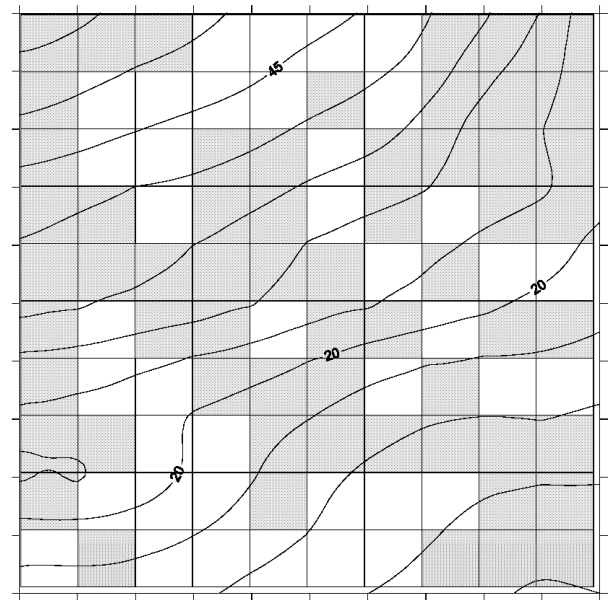

Grupo 1

Grupo 2

Grupo 3

Figura 7. Distribución espacial de los grupos formados en los análisis de clasificación cuando se consideró como variable: (a) presencia o ausencia de especies, (b) densidad de cada especie y (c) logaritmo natural del área basal de cada especie.
Cuadro 3. Índices de diversidad de la parcela calculados para diferentes tamaños de DAP. Para el índice $\alpha$ de Fisher se muestra entre paréntesis el valor de $\mathrm{x}$, que en todos los casos es mayor que 0.5.

\begin{tabular}{cccccc}
\hline $\begin{array}{c}\text { DAP } \\
(\mathrm{cm})\end{array}$ & $\mathrm{H}^{\prime}$ & $\mathrm{H}^{\prime}$ max & $\mathrm{E}$ & $\alpha$ de Fisher & $\begin{array}{c}\text { Índice de } \\
\text { Berger-Parker }\end{array}$ \\
\hline$\geq 2.5$ & 3.99 & 5.29 & 0.75 & $8.081(0.99)$ & 0.14 \\
$\geq 3.3$ & 4.02 & 5.21 & 0.77 & $8.022(0.99)$ & 0.17 \\
$\geq 5$ & 3.82 & 5.04 & 0.76 & $7.512(0.99)$ & 0.21 \\
$\geq 10$ & 3.51 & 4.81 & 0.73 & $6.568(0.99)$ & 0.26 \\
$\geq 15$ & 3.29 & 4.64 & 0.71 & $6.107(0.98)$ & 0.32 \\
$\geq 20$ & 3.11 & 4.52 & 0.69 & $5.866(0.98)$ & 0.33 \\
\hline
\end{tabular}

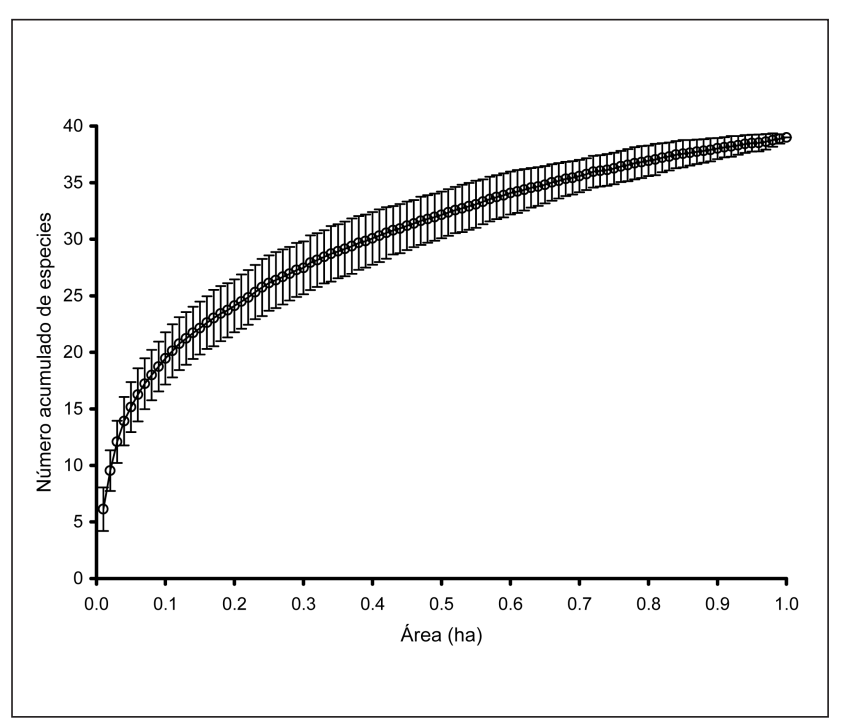

Figura 8. Curva de acumulación de especies con relación al área muestreada. Se muestran las medias correspondientes a cada incremento de 0.01 ha, las barras de error son \pm 1 D.E. La curva fue suavizada por medio de la generación de 500 curvas basadas en la determinación aleatoria de la secuencia de los cuadros de $100 \mathrm{~m}^{2}$.

el número de 48 especies estimadas con el modelo de Jackknife tal vez esté más cercano al número real de especies en este bosque.

\section{Discusión}

Heterogeneidad interna de la parcela. En la parcela de estudio se distinguen al menos dos condiciones topográficas contrastantes: las esquinas $\mathrm{NO}$ y $\mathrm{SO}$ corresponden a las laderas bajas de una cañada, mientras que el resto de la 
parcela se localiza más bien sobre una cima. A pesar de que no se midieron variables ambientales en estos sitios, durante el trabajo de campo fue posible percibir que en las porciones bajas de la cañada, la humedad del aire y del suelo eran mayores que en la zona más alta de la parcela, donde la insolación alcanzaba una mayor intensidad. Sin embargo, esta heterogeneidad topográfica no se reflejó en la estructura de la vegetación de la parcela. Los grupos de cuadros de $100 \mathrm{~m}^{2}$ que se formaron a partir del análisis de clasificación quedaron entremezclados en la parcela, en lugar de separarse espacialmente, como se esperaba. Esta aparente ausencia de estructura espacial contrasta con los patrones de distribución de algunas especies que sí parecen ser sensibles a la variación topográfica. Por ejemplo, los individuos de Berberis moranensis, Fuchsia arborescens, Meliosma dentata, Myrcianthes fragans, Orepanax langlassei, Persea americana, Prunus brachybotrya, P. serotina var. capuli, Sapium sp., Trichilia havanensis, Vallesia aurantiaca, Viburnum membranaceum y Zinowiewia sp. sólo están presentes en las zonas bajas ubicadas en la cañada. En contraste, Cleyera theaeoides, Quercus candicans, $Q$. glabrescens, $Q$. ocoteifolia, $Q$. uxoris y $Q$. skinneri aparecieron preferentemente en las zonas más secas de la parcela. Incluso se encontraron asociaciones negativas entre pares de especies que se distribuyen en agregados en alguna de estas condiciones topográficas, como el caso de Cleyera theaeoides y Zinowiewia sp.

Diversos estudios han mostrado que los patrones de distribución espacial pueden estar afectados por los procesos de regeneración y dispersión de las especies (MartínezRamos, 1994; Richards, 1996). Éste podría ser el caso de Psychotria galeottiana y Miconia sp. nov., para las que se detectaron patrones de distribución agregados, así como una mutua asociación positiva, ya que su distribución parece estar relacionada con las áreas de baja densidad de grandes copas del dosel superior. El análisis de la estructura vertical mostró que el dosel superior se distingue claramente del inferior; es posible también que esta fuerte estratificación sea resultado del régimen de disturbio caracterizado principalmente por la caída de árboles y ramas grandes (Grubb y Tanner, 1976), fenómeno observado con frecuencia durante el trabajo de campo.

En el bosque de Teipan, la dominancia se concentra en dos especies del dosel superior, Cornus disciflora y Quercus ocoteifolia. Este rasgo distingue a los bosques húmedos de montaña de la vegetación tropical de tierras bajas, en donde generalmente las especies dominantes pertenecen al sotobosque y cuya dominancia se debe a su alta densidad (Meave, 1990; Meave et al., 1992).

La presencia de individuos policaulescentes en Teipan, la mayoría de los cuales sólo alcanzan tallas pequeñas, también podría estar relacionada con la frecuencia de caída de árboles y ramas grandes. En estos individuos, la capacidad de rebrote después de sufrir daño físico resulta en la presen- cia de muchos tallos unidos en la base del individuo. En realidad, la policaulescencia es común en este tipo de vegetación (Valencia y Jørgensen, 1992; Valencia, 1995; Arellanes-C., 2000), pero no existe una única explicación satisfactoria para este fenómeno. Si bien hay indicios de que su presencia está relacionada con la altitud (Valencia y Jørgensen, 1992; Valencia, 1995), en bosques tropicales secos de tierras bajas la policaulescencia también llega a ser frecuente, particularmente si la vegetación está expuesta a disturbios más o menos intensos (Dunphy et al., 2000).

Comparación con otros bosques tropicales montanos de niebla. La localización de Teipan prácticamente en el borde de una región de bosque montano de niebla condujo a la pregunta de qué tanto difería este bosque de otros similares, y en cuál o cuáles atributos se concentraban estas posibles diferencias.

El análisis de las variables estructurales generales para la parcela no fueron consistentes respecto a esta pregunta. Por ejemplo, el valor de área basal calculado en Teipan supera los valores medios del intervalo conocido para los bosques tropicales montanos de niebla de América tropical. En contraste, la densidad total es coincidente con los valores reportados para los "upper montane rain forests" (UMRF; bosques lluviosos montanos altos) y de hecho es uno de los valores más bajos (apéndice 1).

Tanto la altura general del dosel como la altura máxima registradas en Teipan también caen dentro de los intervalos conocidos para los llamados "lower montane rain forests" (LMRF; bosques montanos lluviosos bajos) de América tropical, y son mucho más grandes que los valores de altura conocidos para los UMRF (apéndice 1). Respecto a la cobertura, la comparación sólo se hizo con pocos sitios, la mayoría de ellos ubicados en México, ya que son los únicos que consignan este dato. En Teipan se obtuvo un valor de cobertura similar al reportado por Arellanes-C. (2000) para Tiltepec, Sierra Norte de Oaxaca, y casi del doble del valor reportado para Omiltemi (Meave et al., 1992); asimismo, la cobertura es muy superior al valor obtenido en Simojovel, Chiapas (Zuill y Lathrop, 1975), aunque es intermedio entre los correspondientes a dos ambientes contrastantes (ladera E y ladera O) en Puerto Soledad, Oaxaca (Ruiz-Jiménez et al., 2000).

En síntesis, y de acuerdo con estas comparaciones, se puede concluir que ni el área basal, ni la cobertura, ni la altura del dosel reflejan la condición marginal del bosque de Teipan. De hecho, estos valores lo hacen indistinguible de los LMRF del continente, y sólo la baja densidad, valor ubicado en el límite inferior del intervalo conocido, parece reflejar la condición marginal ecológica de este bosque.

A diferencia de la comparación estructural, el contraste de la diversidad del bosque de Teipan y otros bosques sí dejó entrever algunas diferencias notables. En términos del valor $\alpha$ de Fisher y de la riqueza, este bosque ocupa una de 
las posiciones más bajas, ya que sólo supera a los valores reportados para Pasochoa, Ecuador (Valencia y Jørgensen, 1992), los remanentes de bosque mesófilo en el centro de Veracruz (Williams-Linera et al., 1996) y el bosque de Omiltemi, Guerrero (Meave et al., 1992). Pasochoa es el sitio con mayor altitud $(3,300 \mathrm{~m})$ de los incluidos en la comparación, por lo que hubiéramos esperarado a priori que tuviera una menor diversidad, de acuerdo a las tendencias generales descritas para gradientes altitudinales en montañas tropicales (Gentry, 1988). Por ello, llama la atención que Teipan supera en diversidad a Omiltemi, sitio ubicado también en la Sierra Madre del Sur (México). Si bien la menor diversidad registrada en Omiltemi podría explicarse en términos de su precipitación relativamente baja, en realidad sus valores de diversidad son parecidos y ambos se encuentran entre los más bajos reportados para los bosques húmedos de montaña. Si futuros estudios permiten generalizar este patrón, se podría concluir que los bosques mesófilos de montaña de la Sierra Madre del Sur son los más pobres del país en cuanto a su florística.

Las familias presentes en la parcela estudiada han sido reportadas con frecuencia para los bosques tropicales húmedos de montaña de América de los que se dispone de información estructural (Meave et al., 1992; Valencia y Jørgensen, 1992; Madsen y Øllgaard, 1993; Jørgensen et al., 1995; Valencia, 1995; Lieberman et al., 1996; Monedero, 1998; Smith y Killeen, 1998; Arellanes-C., 2000). Tomando en cuenta la escala de estudio (una hectárea), Teipan comparte un mayor número de géneros y especies con áreas iguales de bosque mesófilo ubicadas en la Sierra Madre del Sur. Por ejemplo, con el bosque de Manantlán (Jalisco) comparte 21 géneros y 7 especies; con Omiltemi (Guerrero), 16 géneros y 6 especies, y con ambos comparte 15 géneros: Clethra, Cornus, Meliosma, Miconia, Oreopanax, Parathesis, Prunus, Quercus, Saurauia, Styrax, Ternstroemia, Trichilia, Turpinia, Viburnum y Zinowiewia. En contraste, la estructura florística de este bosque sólo comparte tres o cuatro géneros con los bosques estudiados ubicados en la Sierra Norte de Oaxaca y en Chiapas. En conclusión, la composición florística arbórea de la parcela estudiada en Teipan es más afín a la composición de otras parcelas ubicadas en la Sierra Madre del Sur.

Las familias, los géneros y las especies presentes en la parcela de Teipan son exclusivas, casi exclusivas o al menos muy frecuentes en los bosques mesófilos de montaña de México en general (Rzedowski, 1996). Sin embargo, es notable que los encinares secos colindantes con este bosque también parecen enriquecer su composición, ya que cerca de $18 \%$ de las especies registradas en la parcela también han sido recolectadas en dichos bosques (C.A. RuizJiménez, datos no publicados). En la misma región donde está ubicado Teipan, pero a menores altitudes, existen grandes áreas de vegetación tropical estacionalmente seca (selva baja caducifolia, sensu Miranda y Hernández-X., 1963), pero ninguna de las especies de esas comunidades parece tolerar las condiciones húmedas y frías de las áreas de alta montaña, como lo sugiere su ausencia en el bosque mesófilo. Esta situación contrasta fuertemente con la que tiene lugar en regiones más húmedas, donde los bosques de montaña colindan en sus partes bajas con vegetación forestal de carácter más mésico; este tipo de contactos podría resultar en un enriquecimiento de la composición de las comunidades de montaña a partir de la incorporación de elementos florísticos propios de las tierras bajas (Cartujano et al., 2002). Sería deseable que futuros estudios examinen con más detalle esta posibilidad.

\section{Agradecimientos}

Extendemos un sincero agradecimiento al pueblo y las autoridades de Teipan, en particular al Sr. Javier Ramírez, por su apoyo incondicional para la realización de este trabajo. Leticia Merino e Irene Pisanty invitaron a los autores a colaborar en el proyecto de Teipan y su generosidad permitió canalizar recursos del proyecto DGAPA-IN 309799 para financiar este estudio y para otorgar una beca a la primera autora.

\section{Literatura citada}

Acosta C.S. 1997. Afinidades fitogeográficas del bosque mesófilo de montaña de la zona de Pluma Hidalgo, Oaxaca, México. Polibotánica 6:25-39.

Alder D. y Synnott T.J. 1992. Permanent Sample Plot Techniques for Mixed Tropical Forest. Tropical Forestry Papers 25. Oxford Forestry Institute, Oxford.

Anónimo. 1981. Atlas Nacional del Medio Físico. Secretaría de Programación y Presupuesto, Dirección General de Geografía del Territorio Nacional, México, D.F.

Arellanes-C. Y. 2000. Análisis estructural de un bosque mesófilo de montaña de Ticodendron incognitum en la Sierra Norte de Oaxaca, México. Tesis de Licenciatura, Facultad de Ciencias, Universidad Nacional Autónoma de México, México, D.F., 83 pp.

Beard J.S. 1955. The classification of tropical American vegetation types. Ecology 36:89-100.

Bongers F. 2001. Methods to assess tropical rain forest canopy structure: an overview. Plant Ecology 153:263-277.

Campos-Villanueva A. y Villaseñor J.L. 1995. Estudio florístico de la porción central del municipio de San Jerónimo Coatlán, Distrito de Miahuatlán (Oaxaca). Boletín de la Sociedad Botánica de México 56:95-120.

Cartujano S., Zamudio S., Alcántara O. y Luna I. 2002. El bosque mesófilo de montaña en el municipio de Landa de Matamoros, Querétaro, México. Boletín de la Sociedad Botánica de México 70:13-43.

Colwell R.K. 2001. EstimateS V6.0b1. Statistical Estimation of Species Richness and Shared Species from Samples. http://viceroy.eeb.uconn.edu/estimates.

Cox W.J. 1993. Laboratory Manual of General Ecology. 7a. ed., 
San Diego State University, San Diego.

Dunphy B.K., Murphy P.G. y Lugo A.E. 2000. The tendency for trees to be multiple-stemmed in tropical and subtropical dry forest: Studies of Guanica forest, Puerto Rico. Tropical Ecology 41:161-167.

Ferrusquía-Villafranca J. 1998. Geología de México: una sinopsis. En: Ramamoorthy T.P., Bye R., Lot A. y Fa J. Eds. Diversidad Biológica de México: Orígenes y Distribución, pp. 3-108, Instituto de Biología, Universidad Nacional Autónoma de México, México, D.F.

García de Miranda E. 1989. Apuntes de Climatología. Editado por la autora, México, D.F.

Gentry A.H. 1988. Changes in plant community diversity and floristic composition on environmental and geographical gradients. Annals of the Missouri Botanical Garden 75:1-34.

Goldsmith F.B., Harrison C.M. y Morton A.J. 1976. Description and analysis of vegetation. En: Moore P. y Chapman S.B. Eds. Methods in Plant Ecology, pp. 437-521, Blackwell Scientific Publications, Oxford.

Grubb P.J. y Tanner V.J. 1976. The montane forest and soils of Jamaica: a reassessment. Journal of the Arnold Arboretum 57:313-368.

Hamilton L.S., Juvik J.O. y Scatena F.N. 1995. The Puerto Rico tropical cloud forest symposium: Introduction and workshop synthesis. En: Hamilton L., Juvik J.O. y Scatena F.N. Eds. Tropical Montane Cloud Forest, pp. 1-23, Springer-Verlag, Nueva York.

Hayek L.C. y Buzas M.A. 1997. Surveying Natural Populations. Columbia University Press, Nueva York.

Heany A. y Proctor J. 1990. Preliminary studies on forest structure and floristics on Volcan Barva, Costa Rica. Journal of Tropical Ecology 6:307-320.

IG-UNAM (Instituto de Geografía, Universidad Nacional Autónoma de México). 1970. Carta de Climas. 1:500,000. Oaxaca. 14Q-VIII. Instituto de Geografía, Universidad Nacional Autónoma de México, México, D.F.

INEGI. 1984. Carta de efectos climáticos regionales, mayooctubre. 1:250,000. Juchitán E15-10, D15-1. Instituto Nacional de Estadística, Geografía e Informática. México, D.F.

INEGI. 1989. Carta Geológica. 1:250,000. Juchitán E15-10, D151. Instituto Nacional de Estadística, Geografía e Informática. México, D.F.

Jørgensen P.M., Ulloa U.C., Madsen J.E. y Valencia R.R. 1995. A floristic analysis of the high Andes of Ecuador. En: Churchill S.P., Balslev H., Forero M. y Luteyn J.L. Eds. Biodiversity and Conservation of Neotropical Forests, pp. 221-237, The New York Botanical Garden Press, Nueva York.

Kappelle M. 1996. Los Bosques de Roble (Quercus) de la Cordillera de Talamanca, Costa Rica. Biodiversidad, Ecología, Conservación y Desarrollo. Instituto de Biodiversidad y Universidad de Amsterdam, Wageningen.

Krebs C.J. 1978. Ecology. The Experimental Analysis of Distribution and Abundance. Harper Collins, Nueva York.

Lieberman D., Lieberman M., Peralta R. y Hartshorn G. 1996. Tropical forest structure and composition on a large-scale altitudinal gradient in Costa Rica. Journal of Ecology 84:137-152.

Madsen J.E. y Øllgaard B. 1993. Floristic composition, structure and dynamics of an upper montane rain forest in southern Ecuador. Nordic Journal of Botany 14:403-423.
Magurran A.E. 1988. Ecological Diversity and its Measurement. Princeton University Press, Princeton.

Martínez-Ramos M. 1994. Regeneración natural y diversidad de especies arbóreas en selvas húmedas. Boletín de la Sociedad Botánica de México 54:179-224.

Matteucci S.D. y Colma A. 1982. Metodología para el Estudio de la Vegetación. Organización de los Estados Americanos, Washington, D.C.

Meave J. 1990. Estructura y Composición de la Selva Alta Perennifolia de los Alrededores de Bonampak. Instituto Nacional de Antropología e Historia, México, D.F.

Meave J., Soto M., Calvo L., Paz H. y Valencia S. 1992. Análisis sinecológico del bosque mesófilo de montaña de Omiltemi, Guerrero. Boletín de la Sociedad Botánica de México 52:31-77.

Miranda F. y Hernández-X. E. 1963. Los tipos de vegetación de México y su clasificación. Boletín de la Sociedad Botánica de México 28:29-179.

Monedero C. 1998. Quantitative analysis of the arboreal structure in a tropical cloud forest: ramal interior of the Cordillera de la Costa, Loma de Hierro (Estado Aragna), Venezuela. En: Dallmeier F. Ed. Forest Biodiversity in North, Central and South America, and the Caribbean: Research and Monitoring, pp. 427-447, Man and the Biosphere Series, vol. 21. Parthenon, Nueva York.

Popma J., Bongers F. y Meave del Castillo J. 1988. Patterns in the vertical structure of the tropical lowland rain forest of Los Tuxtlas, Mexico. Vegetatio 74:81-91.

Puig H., Bracho R. y Sosa V. 1983. Composición florística y estructura del bosque mesófilo de montaña de Gómez Farías, Tamaulipas, México. Biótica 8:339-359.

Richards P.W. 1996. The Tropical Rain Forest: An Ecological Study. 2a. ed., Cambridge University Press, Cambridge.

Ruiz-Jiménez C.A., Meave J. y Contreras-Jiménez J.L. 2000. El bosque mesófilo de la región de Puerto Soledad (Oaxaca), México: análisis estructural. Boletín de la Sociedad Botánica de México 65:23-67.

Rzedowski J. 1978. Vegetación de México. Limusa, México, D.F.

Rzedowski J. 1996. Análisis preliminar de la flora fanerogámica de los bosques mesófilos de montaña de México. Acta Botanica Mexicana 35:25-44.

Sánchez-Rodríguez E.V., López-Mata L., García-Moya E. y Cuevas-Guzmán R. 2003. Estructura, composición florística y diversidad de especies leñosas de un bosque mesófilo de montaña en la Sierra de Manantlán, Jalisco. Boletín de la Sociedad Botánica de México 73:17-34.

Santiago A.L. y Jardel E.J. 1993. Composición y estructura del bosque mesófilo de montaña en la Sierra de Manantlán, Jalisco-Colima. Biotam 5:13-26.

Smith D.N. y Killeen T.J. 1998. A comparison of the structure and composition of montane and lowland tropical forest in the Serranía Pilón Lajas, Beni, Bolivia. En: Dallmeier F. Ed. Forest Biodiversity in North, Central and South America, and the Caribbean: Research and Monitoring, pp. 681-701, Man and the Biosphere Series, vol. 21, Parthenon, Nueva York.

STATSOFT INC. 1998. STATISTICA for Windows. Manual Ver. 6.0., Tulsa, Oklahoma.

Tanner E.V.J. 1977. Four montane rain forests of Jamaica: A quantitative characterization of the floristics, the soils and the foliar mineral levels, and a discussion of the interrelations. 
Journal of Ecology 65:883-918.

Valencia R.R. 1995. Composition and structure of an Andean forest fragment in eastern Ecuador. En: Churchill S.P., Balslev H., Forero M. y Luteyn J.L. Eds. Biodiversity and Conservation of Neotropical Forests, pp. 239-249, The New York Botanical Garden Press, Nueva York.

Valencia R. y Jørgensen P.M. 1992. Composition and structure of a humid montane forest and the Pasochoa Volcano, Ecuador. Nordic Journal of Botany 12:239-247.

van Tongeren O.F.R. 1995. Cluster analysis. En: Jongman R.H.G., Ter Braak C.J.F. y van Tongeren O.F.R. Eds. Data Analysis in Community and Landscape Ecology, pp. 174-212, Cambridge University Press, Cambridge.

Whitmore T.C. 1975. Tropical Rain Forest of the Far East. Clarendon Press, Oxford.

Fecha de recepción: 25 de marzo de 2004

Versión corregida: 28 de abril de 2004

Aceptado: 30 de abril de 2004
Williams-Linera G. 1991. Nota sobre la estructura del estrato arbóreo del bosque mesófilo de montaña en los alrededores del campamento "El Triunfo", Chiapas. Acta Botanica Mexicana 13:1-7.

Williams-Linera G., Pérez-García I. y Tolome J. 1996. El bosque mesófilo de montaña y un gradiente altitudinal en el centro de Veracruz, México. La Ciencia y El Hombre 23:149-161.

Zar J.H. 1999. Biostatistical Analysis. 4a ed. Prentice Hall, Upper Saddle River.

Zuill H.A. y Lathrop E.W. 1975. The structure and climate of tropical montane forest and an associated pine-oak-liquidambar forest in the northern highlands of Chiapas, Mexico. Anales del Instituto de Biología de la Universidad Nacional Autónoma de México, Serie Botánica 46:73-118. 
Bosque Mesófilo de montaña en la Sierra Madre del Sur

Apéndice 1. Datos estructurales de bosques tropicales de niebla. Para los estudios con área de muestreo $<1$ ha se reportan los datos extrapolados a esta superficie. El valor de $\alpha$ de Fisher se calculó para los trabajos que reportan datos de densidad y número de especies, y se muestran sólo los valores significativos. Para la altura del dosel, $\overline{\mathrm{x}}=$ media y entre paréntesis se indica la altura máxima.

\begin{tabular}{|c|c|c|c|c|c|c|c|c|}
\hline Sitio & $\begin{array}{l}\text { DAP } \\
(\mathrm{cm})\end{array}$ & $\begin{array}{l}\text { Densidad } \\
\text { (ind. ha-1) }\end{array}$ & $\begin{array}{c}\text { Área basal } \\
\left(\mathrm{m}^{2} \mathrm{ha}^{-1}\right)\end{array}$ & $\begin{array}{l}\text { Altura del } \\
\text { dosel }(m)\end{array}$ & $\begin{array}{c}\text { Cobertura } \\
\left(\mathrm{m}^{2} \mathrm{ha}^{-1}\right)\end{array}$ & $S$ & $\begin{array}{l}\alpha \text { de } \\
\text { Fisher }\end{array}$ & Referencia \\
\hline $\begin{array}{l}\text { BMM (1 ha) } \\
\text { Teipan, México } \\
16^{\circ} 14^{\prime} \mathrm{N}, 95^{\circ} 57^{\prime} \mathrm{O} \\
2450 \mathrm{~m} \text { s.n.m. } \\
\mathrm{T}=12.3 \mathrm{P}=1400 \text { ? }\end{array}$ & $\begin{array}{l}\geq 2.5 \\
\geq 3.3 \\
\geq 5 \\
\geq 10 \\
\geq 15 \\
\geq 20\end{array}$ & $\begin{array}{c}1035 \\
821 \\
626 \\
468 \\
369 \\
295\end{array}$ & $\begin{array}{l}46.41 \\
42.77 \\
42.62 \\
41.85 \\
40.56 \\
38.87\end{array}$ & $\begin{array}{c}11-30(35) \\
- \\
- \\
- \\
- \\
-\end{array}$ & $\begin{array}{l}442.47 \\
436.10 \\
421.47 \\
392.33 \\
363.32 \\
329.89\end{array}$ & $\begin{array}{l}39 \\
37 \\
33 \\
28 \\
25 \\
23\end{array}$ & $\begin{array}{l}8.08 \\
8.02 \\
7.51 \\
6.57 \\
6.11 \\
5.87\end{array}$ & Este trabajo \\
\hline $\begin{array}{l}\text { BMM (1 ha) } \\
\text { Omiltemi, México } \\
17^{\circ} 35^{\prime} \mathrm{N}, 99^{\circ} 41^{\prime} \mathrm{O} \\
2100 \mathrm{~m} \text { s.n.m. } \\
\mathrm{T}=14.0 \mathrm{P}=1200\end{array}$ & $\geq 3.3$ & 2069 & 49.81 & $18-25(>35)$ & 263.81 & 33 & 5.61 & $\begin{array}{c}\text { Meave et al. } \\
(1992)\end{array}$ \\
\hline $\begin{array}{l}\text { BMM (1 ha) } \\
\text { Tiltepec, México } \\
17^{\circ} 31^{\prime} \mathrm{N}, 96^{\circ} 18^{\prime} \mathrm{O} \\
1640 \text { m s.n.m. } \\
\mathrm{T}=16.9 \mathrm{P}=4000 ?\end{array}$ & $\begin{array}{l}\geq 3.3 \\
\geq 5 \\
\geq 10 \\
\geq 15\end{array}$ & $\begin{array}{c}1738 \\
1272 \\
764 \\
569\end{array}$ & $\begin{array}{l}42.72 \\
41.87 \\
39.73 \\
37.24\end{array}$ & $\begin{array}{c}17-24(30) \\
- \\
- \\
-\end{array}$ & $\begin{array}{c}468.47 \\
- \\
- \\
-\end{array}$ & $\begin{array}{l}52 \\
45 \\
36 \\
29\end{array}$ & $\begin{array}{l}10.45 \\
9.23 \\
7.88 \\
6.48\end{array}$ & $\begin{array}{c}\text { Arellanes-C. } \\
\text { (2000) }\end{array}$ \\
\hline $\begin{array}{l}\text { TLMRF ( } 1 \text { ha) }(2) \\
\text { Volcán Barva, } \\
\text { Costa Rica } \\
10^{\circ} 24^{\prime} \mathrm{N}, 84^{\circ} 00^{\prime} \mathrm{O} \\
1750 \mathrm{~m} \text { s.n.m. } \\
2300 \mathrm{~m} \text { s.n.m. } \\
\mathrm{T}=10-24 \mathrm{P}=5600\end{array}$ & $\begin{array}{l}\geq 10 \\
\geq 10\end{array}$ & $\begin{array}{l}479 * \\
572 *\end{array}$ & $\begin{array}{l}28.16 \\
41.76\end{array}$ & $\begin{array}{l}22-31 \\
20-30\end{array}$ & - & $\begin{array}{l}64 \\
44\end{array}$ & - & $\begin{array}{l}\text { Lieberman } \\
\text { et al. (1996) }\end{array}$ \\
\hline $\begin{array}{l}\text { LMRF (1 ha) }(3) \\
\text { Volcán Barva, } \\
\text { Costa Rica } \\
10^{\circ} 24^{\prime} \mathrm{N}, 84^{\circ} 00^{\prime} \mathrm{O} \\
1500-2600 \mathrm{~m} \text { s.n.m } \\
\mathrm{T}=10-24 \mathrm{P}=4210\end{array}$ & $\begin{array}{l}\geq 10 \\
\geq 10 \\
\geq 10\end{array}$ & $\begin{array}{l}553 \\
448 \\
617\end{array}$ & $\begin{array}{l}29.20 \\
28.60 \\
51.20\end{array}$ & $\begin{array}{l}25-30(38) \\
20-25(35) \\
20-23(32)\end{array}$ & $\begin{array}{l}- \\
- \\
-\end{array}$ & $\begin{array}{l}65^{* *} \\
69^{* *} \\
35^{* *}\end{array}$ & $\begin{array}{c}19.16 \\
22.97 \\
8.10\end{array}$ & $\begin{array}{c}\text { Heaney y } \\
\text { Proctor } \\
\text { (1990) }\end{array}$ \\
\hline $\begin{array}{l}\text { TMCF (1 ha) } \\
\text { Loma de Hierro, } \\
\text { Venezuela } \\
10^{\circ} 8^{\prime} \mathrm{N}, 87^{\circ} 8^{\prime} \mathrm{O} \\
1355 \text { m s.n.m. } \\
\mathrm{T}=20.2 \mathrm{P}=1588\end{array}$ & $\begin{array}{c}\geq 3 \\
\geq 10\end{array}$ & $\begin{array}{c}2457 \\
678\end{array}$ & $\begin{array}{c}46.40 \\
-\end{array}$ & $\begin{array}{c}20-30(42) \\
-\end{array}$ & - & $\begin{array}{l}60 \\
51\end{array}$ & - & $\begin{array}{c}\text { Monedero } \\
\text { (1998) }\end{array}$ \\
\hline $\begin{array}{l}\text { UMRF ( } 1 \text { ha) } \\
\text { Pasochoa, Ecuador } \\
0^{\circ} 28^{\prime} \mathrm{S}, 78^{\circ} 41^{\prime} \mathrm{O} \\
3300 \text { m s.n.m. } \\
\mathrm{T}=13.8 \quad \mathrm{P}=1489.5\end{array}$ & $\begin{array}{c}\geq 5 \\
\geq 10\end{array}$ & $\begin{array}{c}1058 \\
715\end{array}$ & $\begin{array}{l}25.70 \\
24.20\end{array}$ & $\begin{array}{c}5-14(20) \\
-\end{array}$ & - & $\begin{array}{l}32 \\
27\end{array}$ & $\begin{array}{l}6.30 \\
5.57\end{array}$ & $\begin{array}{c}\text { Valencia y } \\
\text { Jørgensen } \\
\text { (1992) }\end{array}$ \\
\hline
\end{tabular}


Nancy R. Mejía-Domínguez, Jorge A. Meave y Carlos A. Ruiz-Jiménez

\begin{tabular}{|c|c|c|c|c|c|c|c|c|}
\hline Sitio & $\begin{array}{l}\text { DAP } \\
(\mathrm{cm})\end{array}$ & $\begin{array}{l}\text { Densidad } \\
\text { (ind. ha-1) }\end{array}$ & $\begin{array}{c}\text { Área basal } \\
\left(\mathrm{m}^{2} \mathrm{ha}^{-1}\right)\end{array}$ & $\begin{array}{l}\text { Altura del } \\
\text { dosel (m) }\end{array}$ & $\begin{array}{c}\text { Cobertura } \\
\left(\mathrm{m}^{2} \mathrm{ha}^{-1}\right)\end{array}$ & $S$ & $\begin{array}{l}\alpha \text { de } \\
\text { Fisher }\end{array}$ & Referencia \\
\hline $\begin{array}{l}\text { UMRF ( } 1 \text { ha }) \\
\text { Cajunama, } \\
\text { Ecuador } \\
4^{\circ} 05^{\prime} \text { S, } 79^{\circ} 10^{\prime} \mathrm{O} \\
2290 \mathrm{~m} \text { s.n.m. } \\
\mathrm{T}=11 \mathrm{P}=3000\end{array}$ & $\begin{array}{l}\geq 5 \\
\geq 10 \\
\geq 15 \\
\geq 20\end{array}$ & $\begin{array}{l}2310 \\
1273 \\
706 \\
375\end{array}$ & $\begin{array}{l}44.00 \\
39.50 \\
32.50 \\
24.60\end{array}$ & $\begin{array}{c}\bar{x}=7.4(16) \\
- \\
- \\
-\end{array}$ & $\begin{array}{l}- \\
- \\
- \\
-\end{array}$ & $\begin{array}{l}75 \\
67 \\
59 \\
50\end{array}$ & $\begin{array}{l}15.38 \\
15.31 \\
15.36 \\
15.58\end{array}$ & $\begin{array}{c}\text { Madsen y } \\
\text { Øllgaard } \\
\text { (1994) }\end{array}$ \\
\hline $\begin{array}{l}\text { UMRF (1 ha) } \\
\text { Yangana, Ecuador } \\
4^{\circ} 28^{\prime} \text { S, } 79^{\circ} 09^{\prime} \mathrm{O} \\
2700 \mathrm{~m} \text { s.n.m. } \\
\mathrm{T}=12 \mathrm{P}=3000\end{array}$ & $\begin{array}{l}\geq 5 \\
\geq 10 \\
\geq 15 \\
\geq 20\end{array}$ & $\begin{array}{c}2090 \\
438 \\
123 \\
45\end{array}$ & $\begin{array}{l}15.30 \\
8.70 \\
4.60 \\
2.70\end{array}$ & $\begin{array}{c}\overline{\mathrm{x}}= \\
5.4(12) \\
- \\
- \\
-\end{array}$ & $\begin{array}{l}- \\
- \\
- \\
-\end{array}$ & $\begin{array}{l}90 \\
59 \\
37 \\
16\end{array}$ & $\begin{array}{c}19.36 \\
18.50 \\
18.28 \\
-\end{array}$ & $\begin{array}{c}\text { Madsen y } \\
\text { Øllgaard } \\
\text { (1994) }\end{array}$ \\
\hline $\begin{array}{l}\text { UMRF ( } 1 \text { ha) } \\
\text { Baeza, Ecuador } \\
0^{\circ} 28^{\prime} \text { S, } 77^{\circ} 54^{\prime} \mathrm{O} \\
2000 \mathrm{~m} \text { s.n.m. } \\
\mathrm{T}=16-17 \mathrm{P}=2320\end{array}$ & $\geq 5$ & 1622 & 45.10 & $\overline{\mathrm{x}}=4.8(30)$ & - & 55 & 11.21 & $\begin{array}{c}\text { Valencia } \\
\text { (1995) }\end{array}$ \\
\hline $\begin{array}{l}\text { UMRF (1 ha) } \\
\text { Lloa, Ecuador } \\
0^{\circ} 50^{\prime} \text { S, } 78^{\circ} 38^{\prime} \text { O } \\
2900 \text { m s.n.m. } \\
\text { T=13.8 P=1489 }\end{array}$ & $\geq 5$ & 764 & 26.90 & $\overline{\mathrm{x}}=9.6(31)$ & - & 39 & 8.70 & $\begin{array}{l}\text { Jørgensen } \\
\text { et al. (1995) }\end{array}$ \\
\hline $\begin{array}{l}\text { BMM (9 cuadros } \\
\text { de } 0.25 \text { ha) } \\
\text { Gómez Farías, Méx. } \\
23^{\circ} 12^{\prime} \mathrm{N}, 99^{\circ} 18^{\prime} \mathrm{O} \\
1850-2150 \mathrm{~m} \text { s.n.m } \\
\mathrm{T}=19.04 \mathrm{P}=2000\end{array}$ & $\geq 15$ & 1169 & 31.55 & $15-25$ & - & 54 & - & $\begin{array}{l}\text { Puig et al. } \\
\text { (1983) }\end{array}$ \\
\hline $\begin{array}{l}\text { BMM (0.1 ha) (12) } \\
\text { Manantlán, Méx. } \\
19^{\circ} 26^{\prime} \mathrm{N}, 103^{\circ} 51^{\prime} \mathrm{O} \\
400-2800 \text { m s.n.m. } \\
\mathrm{T}=12-27 \mathrm{P}=1800\end{array}$ & $\geq 5$ & 715 & $56.7 \pm 14.7$ & $21-35$ & - & 73 & - & $\begin{array}{c}\text { Santiago y } \\
\text { Jardel } \\
\text { (1993) }\end{array}$ \\
\hline $\begin{array}{l}\text { BMM }(0.1 \text { ha) } \\
\text { Veracruz, México } \\
19^{\circ} 44^{\prime} \mathrm{N}, 96^{\circ} 48^{\prime} \mathrm{O} \\
1900 \mathrm{~m} \text { s.n.m. } \\
\mathrm{T}=14.2 \mathrm{P}=1806\end{array}$ & $\geq 5$ & 1340 & 43.60 & $\bar{x}=17.5$ & - & 23 & - & $\begin{array}{l}\text { Williams- } \\
\text { Linera } \\
(1996)\end{array}$ \\
\hline $\begin{array}{l}\text { BMM (s.a.) } \\
\text { Pto. Soledad, Méx. } \\
18^{\circ} 07^{\prime} \mathrm{N}, 96^{\circ} 57^{\prime} \mathrm{O} \\
\text { Ladera N } \\
2500 \text { m n.s.m } \\
\mathrm{T}=16 \mathrm{P}=1419.4\end{array}$ & $\geq 3.18$ & 1101.67 & 87.84 & 20 & 257.11 & - & - & $\begin{array}{l}\text { Ruiz- } \\
\text { Jiménez et } \\
\text { al. (2000) }\end{array}$ \\
\hline
\end{tabular}


Bosque Mesófilo de montaÑa EN LA Sierra MAdRe DEl SuR

\begin{tabular}{|c|c|c|c|c|c|c|c|c|}
\hline Sitio & $\begin{array}{l}\text { DAP } \\
(\mathrm{cm})\end{array}$ & 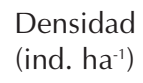 & $\begin{array}{l}\text { Área basal } \\
\left(\mathrm{m}^{2} \mathrm{ha}^{-1}\right)\end{array}$ & $\begin{array}{l}\text { Altura del } \\
\text { dosel (m) }\end{array}$ & $\begin{array}{c}\text { Cobertura } \\
\left(\mathrm{m}^{2} \mathrm{ha}^{-1}\right)\end{array}$ & $S$ & $\begin{array}{l}\alpha \text { de } \\
\text { Fisher }\end{array}$ & Referencia \\
\hline $\begin{array}{l}\text { POLF, MRF (s.a.) } \\
\text { Simojovel, México } \\
17^{\circ} 8^{\prime} \mathrm{N}, 92^{\circ} 55^{\prime} \mathrm{O} \\
2000 \mathrm{~m} \text { s.n.m. } \\
\mathrm{T}=17.3 \mathrm{P}=1407\end{array}$ & $\begin{array}{l}\geq 2.54 \\
\geq 2.54\end{array}$ & $\begin{array}{l}933 \\
963\end{array}$ & $\begin{array}{c}63.44 \\
102.01\end{array}$ & $\begin{array}{l}24.26 \\
27.01\end{array}$ & $\begin{array}{c}- \\
84.6\end{array}$ & - & - & $\begin{array}{l}\text { Zuill y } \\
\text { Lathrop } \\
(1975)\end{array}$ \\
\hline $\begin{array}{l}\text { BMM (0.1 ha) } \\
\text { El Triunfo, México } \\
15^{\circ} 43^{\prime} \mathrm{N}, 92^{\circ} 42^{\prime} \mathrm{O} \\
1850-2150 \mathrm{~m} \text { s.n.m } \\
\mathrm{T}=16 \mathrm{P} \geq 4000\end{array}$ & $\geq 5$ & $960 \pm 102$ & $54.5 \pm 12.4$ & 40 & - & $17 ?$ & - & $\begin{array}{l}\text { Williams- } \\
\text { Linera } \\
(1991)\end{array}$ \\
\hline $\begin{array}{l}\text { LMRF (0.0465 ha) } \\
\text { Borja, Ecuador } \\
0^{\circ} 25^{\prime} \text { S, } 77^{\circ} 50^{\prime} \mathrm{O} \\
1710 \text { ? m s.n.m. }\end{array}$ & $\geq 3.3$ & 495 & - & 27 & - & - & - & $\begin{array}{c}\text { Grubb et al. } \\
(1963)\end{array}$ \\
\hline $\begin{array}{l}\text { MRF (s.a.) } \\
\text { Blue Mountains, } \\
\text { Jamaica } \\
18^{\circ} \mathrm{N}, 77^{\circ} \mathrm{O} \\
1745 \mathrm{~m} \text { s.n.m. } \\
\mathrm{T}=20 \mathrm{P}=4000\end{array}$ & $\geq 3.3$ & 4900 & $\begin{array}{l}64.70 \\
65.43 \\
43.35 \\
47.80\end{array}$ & $\begin{array}{c}4-7 \\
8-13 \\
8-13 \\
12-18\end{array}$ & $\begin{array}{l}- \\
- \\
- \\
-\end{array}$ & $\begin{array}{l}16 \\
34 \\
35 \\
26\end{array}$ & $\begin{array}{l}- \\
- \\
- \\
-\end{array}$ & $\begin{array}{l}\text { Tanner } \\
(1977)\end{array}$ \\
\hline
\end{tabular}

$\mathrm{S}=$ número de especies, ${ }^{*}=$ número de troncos, ${ }^{* *}=$ faltan especies por determinar; s.a. $=$ muestreo sin área. BMM, Bosque mesófilo de montaña; TLMRF, Tropical lower montane rain forest; TMCF, Tropical montane cloud forest; LMRF, Lower montane rain forest; UMRF, Upper montane rain forest; MRF, Montane rain forest; POLF, Pine-Oak-Liquidambar forest; SBP, Selva baja perennifolia. 